\title{
Derivation of parameters for 3748 FGK stars using $H$-band spectra from APOGEE Data Release 14^
}

\author{
Pedro Sarmento ${ }^{1,2}$, Elisa Delgado Mena ${ }^{1}$, Bárbara Rojas-Ayala $^{3}$, and Sergi Blanco-Cuaresma ${ }^{4}$ \\ ${ }^{1}$ Instituto de Astrofísica e Ciências do Espaço, Universidade do Porto, CAUP, Rua das Estrelas, 4150-762 Porto, Portugal \\ e-mail: pedro.sarmento@astro.up.pt \\ 2 Departamento de Física e Astronomia, Faculdade de Ciências, Universidade do Porto, Porto, Portugal \\ 3 Instituto de Alta Investigación, Universidad de Tarapacá, Casilla 7D, Arica, Chile \\ ${ }^{4}$ Harvard-Smithsonian Center for Astrophysics, 60 Garden Street, Cambridge, MA 02138, USA
}

Received 11 July 2019 / Accepted 23 December 2019

\section{ABSTRACT}

\begin{abstract}
Context. The Apache Point Observatory Galactic Evolution Experiment (APOGEE) has observed the $H$-band spectra of over 200000 stars with $R \sim 22000$.

Aims. The main motivation for this work is to test an alternative method to the standard APOGEE pipeline (APOGEE Stellar Parameter and Chemical Abundances Pipeline, ASPCAP) to derive parameters in the near-infrared for FGK dwarfs.

Methods. iSpec and Turbospectrum are used to generate synthetic spectra matching APOGEE observations and to determine the parameters through $\chi^{2}$ minimization.

Results. We present spectroscopic parameters $\left(T_{\mathrm{eff}},[\mathrm{M} / \mathrm{H}], \log g, v_{\mathrm{mic}}\right)$ for a sample of 3748 main-sequence and subgiant FGK stars, obtained from their APOGEE $H$-band spectra.

Conclusions. We compare our output parameters with the ones obtained with ASPCAP for the same stellar spectrum, and find that the values agree within the expected uncertainties. A comparison with the optical samples California Planet Survey, High Accuracy Radial Velocity Planet Searcher - Guaranteed Time Observations, and PASTEL is also available, and median differences below $10 \mathrm{~K}$ for $T_{\text {eff }}$ and $0.2 \mathrm{dex}$ for $[\mathrm{M} / \mathrm{H}]$ are found. Reasons for these differences are explored. The full $H$-band line list, the line selection for the synthesis, and the synthesized spectra are available at the CDS, as are the calculated parameters and their estimated uncertainties.
\end{abstract}

Key words. stars: fundamental parameters - stars: solar-type - techniques: spectroscopic

\section{Introduction}

The analysis of light spectra from stars originated in Newton (1672), and has steadily evolved since then, with the recognition of absorption lines and spectral features greatly aiding our understanding and classification of stars. As instruments and analysis improved over time, spectroscopy has cemented its place as the method to determine physical properties of stellar atmospheres. Spectroscopic stellar parameters, such as effective temperature $T_{\text {eff }}$, stellar composition (metallicity, $[\mathrm{M} / \mathrm{H}]$ ), and surface gravity $(\log g)$, can be determined through an analysis of stellar spectra (Gray 2005). Spectroscopic parameters have been proven to be useful in deriving reliable masses and radii of stars when combined with evolutionary models (Girardi et al. 2002; Pietrinferni et al. 2004; Dotter et al. 2008). The determination of these parameters can therefore provide important data for the understanding and study of stellar evolution (e.g., Girardi et al. 2000), galactic history (e.g., Cunha et al. 2016), star selection for planet detection surveys (e.g., Fleming et al. 2015), and characterization of known planet host stars (e.g., Bean et al. 2006; Santos et al. 2013). Spectroscopy can help to characterize each star's present conditions, to infer its past conditions, and to

\footnotetext{
* The line list, line mask, and parameter table are only available at the CDS via anonymous ftp to cdsarc.u-strasbg. fr (130.79.128.5) or via http://cdsarc.u-strasbg.fr/viz-bin/cat/J/A+A/636/ A85
}

understand the formation environments and galactic populations (e.g., Koleva et al. 2008; Gazzano et al. 2010).

Due to the importance of spectroscopic stellar parameters for the proper characterization of stars, there has been an increase in the number of spectroscopic surveys to characterize FGK stars in the solar neighborhood (e.g., Sousa et al. 2008; Adibekyan et al. 2012; Tsantaki et al. 2013; Bensby et al. 2014; Brewer et al. 2016), and of larger surveys that observe deeper into the Milky Way, such as the Gaia-ESO Survey (Gilmore et al. 2012), Galactic Archaeology with HERMES (GALAH, De Silva et al. 2015), and the Large sky Area Multi-Object fiber Spectroscopic Telescope (LAMOST, Zhao et al. 2012). Most of these studies are focused on spectra observed within visible wavelengths $(400 \mathrm{~nm})$. However, probing other sections of the electromagnetic spectrum for the derivation of stellar parameters can give us another perspective on stellar characterization through spectroscopy, and can provide a comparison for stellar parameters derived from optical spectra. The near-infrared (NIR) wavelengths, in particular, allow us to perform observations beyond the large molecular clouds in the galaxy, as visual extinction is lower in that wavelength range. As FGK stars are still relatively bright in this wavelength range and have features that can be used to characterize them, their NIR spectra can be useful to analyze and characterize these types of stars. Lower temperature stars, like $\mathrm{M}$ dwarfs and red giants, have less complex spectra and are brighter in the NIR than in optical wavelengths and allow 
us to find more precise and accurate spectroscopic parameters (e.g., Önehag et al. 2012; Rojas-Ayala et al. 2012).

We have arrived at the era of instruments that provide highresolution spectra in the NIR, such as CARMENES $(R=$ 80 000-100 000, Quirrenbach et al. 2014), GIANO ( $R \sim 50000$, Origlia et al. 2014), and SPIROU ( $R \sim 75000$, Artigau et al. 2014), as well as large surveys that provide medium-resolution NIR spectra for thousands of stars, like the Apache Point Observatory Galactic Evolution Experiment (APOGEE, Allende Prieto et al. 2008). APOGEE is an $H$-band (1.5-1.7 micron) Sloan Digital Sky Survey program that focuses on obtaining $R \sim 22500$ stellar spectra with a 300-fiber spectrograph. It is split between APOGEE-N, using the Sloan $2.5 \mathrm{~m}$ telescope at the Apache Point Observatory in New Mexico (Gunn et al. 2006), and APOGEE-S, which uses the $2.5 \mathrm{~m}$ duPont telescope at the Las Campanas Observatory in Chile (Bowen \& Vaughan 1973). It targets mostly red giants and provides public spectra for more than 200000 stars in its latest Data Release (DR14, Holtzman et al. 2018). In addition to these stars, APOGEE has observed FGK and M dwarfs for calibration purposes or as part of ancillary programs. Parameters for these stars have been derived with APOGEE Stellar Parameter and Chemical Abundances Pipeline (ASPCAP, Garcia Pérez et al. 2016). ASPCAP works by searching and interpolating a grid of synthetic spectra to find the best match for each observed spectrum, adopting the parameters of the best match as the best parameters for each star. These parameters are then calibrated to best match the theoretical models.

In this paper, we provide an alternative methodology to derive spectroscopic parameters using spectral synthesis in the $H$-band spectra of FGK stars observed by APOGEE and tested its results against similar techniques performed in the optical. Our goal is to have a reliable spectroscopic method for FGK star characterization in the $H$-band obtained with current and future medium and high-resolution $H$-band spectrographs.

The remainder of this paper is structured as follows: details of our object sample and their literature pertinent to our study are described in Sect. 2. The methodology, including the preparation of the APOGEE spectra and all the steps required to obtain stellar parameters from them, are explained in Sect. 3. Section 4 presents both the spectra and parameters derived for solar-type stars, including a visual comparison between the synthetic and the normalized spectra for selected stars. Section 5 includes the analysis and comparison of the derived parameters to different literature sources, while possible sources of discrepancies are explored. Finally, in Sect. 6, we summarize our conclusions regarding our pipeline and its ability to provide accurate and precise parameters for the Sun and solar-type stars from their APOGEE spectra.

\section{Data}

Our complete sample consists of 3748 stars with $H$-band spectra from the APOGEE survey Data Release 14 (Holtzman et al. 2018). All of these stars have spectroscopic parameters derived with the APOGEE Stellar Parameter and Chemical Abundances Pipeline (ASPCAP, Garcia Pérez et al. 2016). The main goal of ASPCAP is to provide parameters for giant stars, but values for dwarf stars are published as well. The spectroscopic parameters and chemical abundances are determined by ASPCAP in a two step fashion. First, to derive atmospheric parameters such as $T_{\text {eff }},[\mathrm{M} / \mathrm{H}]$, and $\log g$, APOGEE observations are compared to a large library of synthetic spectra to find the spectrum that best matched the observed one by interpolating different synthetic models. This library is separated into five smaller

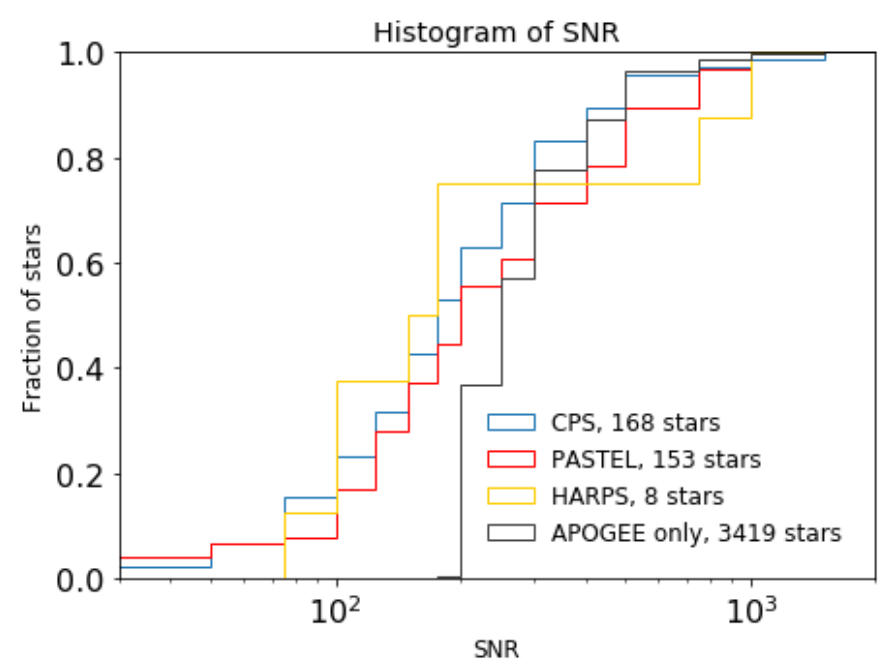

Fig. 1. Cumulative histogram of the APOGEE-published $\mathrm{S} / \mathrm{N}$ of our stellar sample.

sections, for GK dwarfs, GK giants, $M$ dwarfs, $M$ giants, and F-type stars. Secondly, to derive the abundance of individual elements, the atmospheric parameters obtained from the best fit synthetic spectrum are used to fit limited regions of the spectra dominated by spectral features associated with each given element. Afterwards, $T_{\text {eff }}$ and $[\mathrm{M} / \mathrm{H}]$ values for dwarf stars are both calibrated using independent methods to ensure their accuracy. $T_{\text {eff }}$ values were calibrated by minimization of the differences between ASPCAP and photometric observations by Hernandez \& Bonifacio (2009). [M/H] values were internally calibrated as a function of $T_{\text {eff }}$. In addition, a zero-point shift was adopted to force the mean abundance ratios of all observed stars with solar metallicity to be zero. Despite finding their $\log g$ values for $\mathrm{M}$ dwarfs to be too low based on expectations from stellar isochrones, due to a lack of a significant number of asteroseismic calibrators available for dwarf stars, ASPCAP does not calibrate them in any way ${ }^{1}$.

Our selection was done by selecting objects with ASPCAP $T_{\text {eff }}$ values between 5500 and $6200 \mathrm{~K}, S / N \geq 200$ and $-0.5 \mathrm{dex}<[\mathrm{M} / \mathrm{H}]<0.5 \mathrm{dex}$. This was done to exclude stars with low-quality spectra and to ensure a sample both homogeneous and with parameters close to solar. A total of 3419 mainsequence stars matching these criteria are in the sample. Additional stars, not meeting these criteria, were included as well as part of subsamples previously observed in the optical. Most of these additional stars are also main-sequence FGK stars, but some of them are turn-off stars, and have therefore lower $\log g$ values than the ones still on the main sequence. These subgiant stars were included for statistical reasons, as the number of FGK main-sequence stars in common between APOGEE and other optical surveys is not large enough for our purposes, and to test the limits of the parameter space. Our primary focus remains on the main-sequence stars. The total of 3748 stars includes those additional stars observed in optical wavelengths. Those stars are described in Sect. 2.2. The APOGEE S/N distribution of our sample of spectra is shown in Fig. 1.

\footnotetext{
1 Exact values and calibrations are available in Holtzman et al. (2018), and more information can also be consulted at the APOGEE website https://www.sdss.org/dr14/irspec/aspcap/
} 


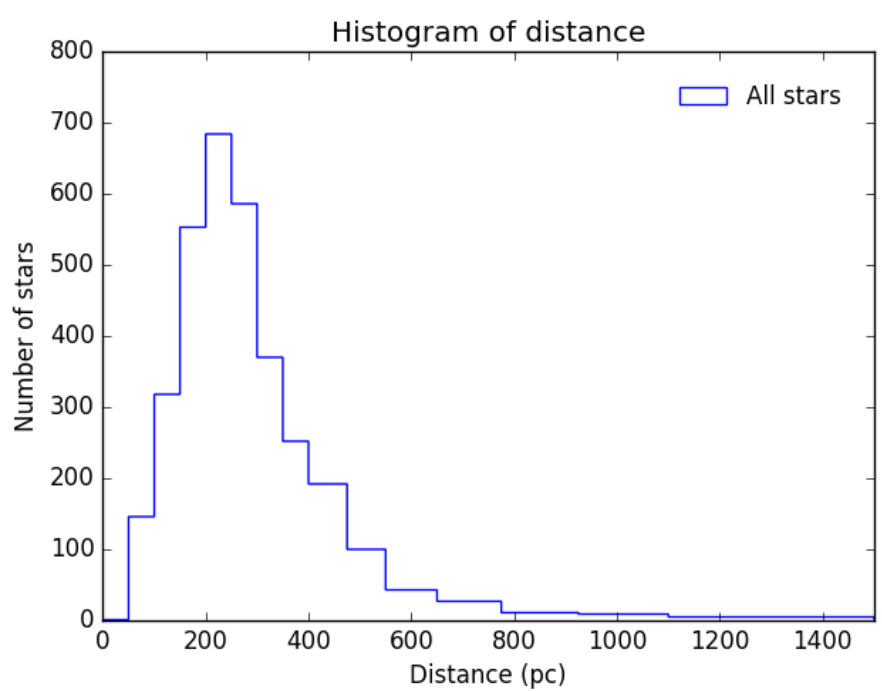

Fig. 2. Histogram of the calculated distances to the sample stars, using Gaia Data Release 2 values for the parallax (Brown et al. 2018).

\subsection{Sample characterization}

Parallaxes, positions, and optical photometry for 3645 stars in our sample were found in the Gaia Data Release 2 (Brown et al. 2018). Due to either inaccurate proper motion measurements or lack of observations from Gaia, no matches were discovered for the remaining 103 stars, but their overall distribution should match that of the other stars. The large majority of the stars are located between $100 \mathrm{pc}$ and $500 \mathrm{pc}$ from us, with only a few reaching distances above $1 \mathrm{Kpc}$ (see Fig. 2). Therefore, our sample, while not entirely composed of solar neighborhood stars $(d<50 \mathrm{pc})$, consists of local stars. Their magnitudes in the Gaia and $H$-band filters are shown in Fig. 3. Most of these objects exhibit magnitudes in the ranges of $9<G<13$ and $8<H<11$. Their distances and magnitudes confirm to us that the sample is composed of FGK dwarf stars, as any giant stars in the sample would either be brighter or further away from us. Their location in the sky is shown in Fig. 4. Given that the majority of the observations belong to APOGEE-N, it is no surprise that most of the stars are located in the northern celestial hemisphere. The concentric circles represent the fields chosen by the APOGEE team for follow-up. In red, 449 stars in our sample that are a part of the Kepler Field (Latham et al. 2005) are shown.

\subsection{Comparison subsamples}

We selected three works in the literature that have analyzed APOGEE stars with medium or high-resolution optical spectra. These stars were added to our full sample, even if they did not meet our selection criteria $\left(5500 \mathrm{~K}<T_{\text {eff }}<6200 \mathrm{~K}, S / N \geq 200\right.$ and $-0.5 \mathrm{dex}<[\mathrm{M} / \mathrm{H}]<0.5 \mathrm{dex})$. This was done so more stars with accurate stellar parameters were available for the comparison of our results.

The California Planet Survey (Brewer et al. 2016, hereby referred to as CPS) used data from the HIRES spectrograph $(R \sim 70000)$ at the Keck Observatory. The stellar parameters were obtained with the semi-automated procedure SME (Valenti \& Piskunov 1996), where the observed spectra were fitted iteratively with synthetic spectra from 1D local thermodynamic equilibrium (LTE) plane-parallel MARCS atmosphere models. CPS provides rotational velocities and abundances for 15 elements as well. The reported precisions are of $25 \mathrm{~K}$ for $T_{\text {eff }}$,

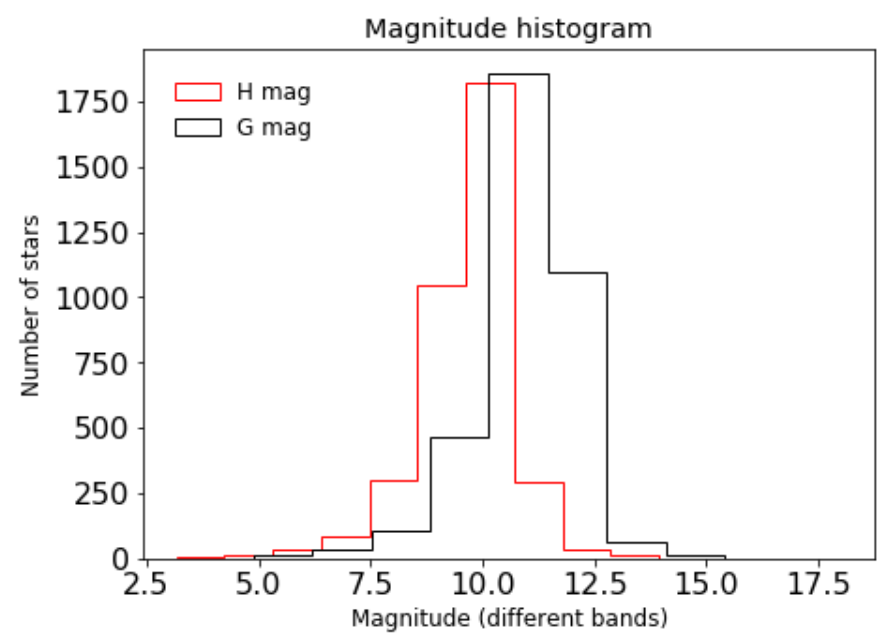

Fig. 3. Histogram of the magnitudes for stars in the sample. $G$ band data comes from Brown et al. (2018); $H$ band from APOGEE.

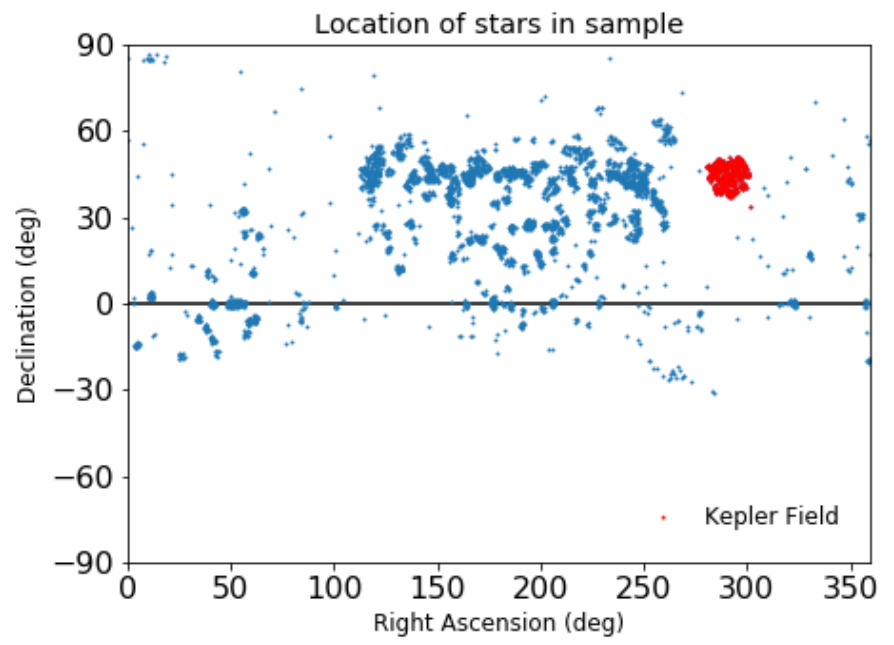

Fig. 4. Map showing the location in the sky (Right Ascension and Declination) of our sample stars.

$0.01 \mathrm{dex}$ for $[\mathrm{M} / \mathrm{H}]$ and $0.028 \mathrm{dex}$ for $\log g$. One-hundred and sixty eight stars in our sample are found in the CPS catalog.

The PASTEL catalog (Soubiran et al. 2016) is a compilation of spectroscopic parameters from high-resolution $(R \geq 25000)$ spectra with $S / N \geq 50$. It contains results from different sources in the literature that derived stellar parameters with model atmospheres. The reported uncertainties vary, with median errors of $\sim 1.1 \%$ for the $T_{\text {eff }}(\sim 65 \mathrm{~K}$ for a sun-like star $), \sim 0.06 \mathrm{dex}$ for $[\mathrm{Fe} / \mathrm{H}]$ and 0.10 dex for $\log g$. A total of 157 stars in our sample are found in the PASTEL catalog. However, as 4 of these stars are also found in CPS, only 153 stars were considered as the PASTEL comparison sample.

Delgado Mena et al. (2017) revised the spectroscopic parameters and abundances of 1111 FGK dwarf stars of the High Accuracy Radial velocity Planet Searcher Guaranteed Time Observations planet search program (HARPS-GTO, Pepe et al. 2000, 2011). The equivalent width (EW) method was used on HARPS-GTO $R \sim 115000$ spectra for parameter and abundance determinations (Sousa et al. 2008; Tsantaki et al. 2013). Their average cited internal errors are $24 \mathrm{~K}$ for $T_{\text {eff }}, 0.02$ dex for $[\mathrm{M} / \mathrm{H}]$ and 0.03 dex for $\log g$. Given that the HARPS-GTO planet survey concentrates on bright inactive stars (mostly $V_{\text {mag }}<11$ ), 
only eight stars in our sample are part of the 1111-star sample characterized in Delgado Mena et al. (2017).

\section{Methodology}

This section describes our method and the steps required to derive parameters from APOGEE's $H$-band spectra.

\section{1. iSpec and Turbospectrum}

iSpec is a multi-purpose python-based tool designed to derive atmospheric parameters from stellar spectra through different methods (Blanco-Cuaresma et al. 2014; Blanco-Cuaresma 2019). Among the radiative transfer codes available to use within iSpec, for spectral synthesis we chose Turbospectrum, developed by Alvarez \& Plez (1998) and Plez (2012). Turbospectrum is the fastest code available within iSpec and has shown to be compatible with our line list.

The code uses a least-squares algorithm to match the two spectra, using the synthetic spectra generator chosen by the user. The code then runs its minimization routine and provides an output that corresponds to the spectrum that best matches the input. It provides an estimate of the errors, based on the spectrum errors, as well as the $\chi^{2}$ value for the best match. These values are included along with our derived parameters for the stars in Table C.1.

\subsection{Stellar models}

iSpec is compatible with two categories of models available: MARCS (Gustafsson et al. 2008) and ATLAS9 (Kurucz 2005). There are some subcategories of models within each of these. We chose the MARCS.GES models for our syntheses since they offer better coverage in the parameter space we are interested in. The MARCS.GES models cover $T_{\text {eff }}$ from $2500 \mathrm{~K}$ to $8000 \mathrm{~K}$ and $\log g$ from $0.0 \mathrm{dex}$ to $5.5 \mathrm{dex}$, allowing us to extend our method to the $\mathrm{M}$ dwarf regime using the same models (Sarmento et al., in prep.). The MARCS.GES models assume plane-parallel 1D stratification, hydrostatic equilibrium, mixing-length convection, and local thermodynamic equilibrium. MARCS.GES also take into account $[\alpha / \mathrm{Fe}]$ and $[\mathrm{O} / \mathrm{Fe}]$ parameters when generating the models. Standard models for $[\mathrm{Fe} / \mathrm{H}]<-0.25$ dex have $[\alpha / \mathrm{Fe}]$ and $[\mathrm{O} / \mathrm{Fe}]$ values tailored to match abundances measured in the solar neighborhood stars.

For the solar abundances we used the values available in Grevesse et al. (2007) as they were the ones used to create the MARCS models used in the synthesis.

\subsection{Normalization}

APOGEE DR14 spectra includes both normalized spectra and multiple stages of spectrum processing for its stars ${ }^{2}$. The normalized spectra are processed by ASPCAP to derive parameters and could, in principle, be used to fit individual lines and determine abundances and parameters using the EW method, for example. However, the normalization done for APOGEE DR14 spectra is not precise enough to compare the normalized spectrum to our full synthetic spectrum. Therefore, we had to perform our own normalization to the combined spectrum ${ }^{3}$ of each star in our sample.

\footnotetext{
2 https://www.sdss.org/dr14/data_access/

3 https://data.sdss.org/sas/dr14/apogee/spectro/redux/ r8/stars/
}

Our normalization method relies on what we call a reference spectrum. We created a grid of normalized synthetic spectra covering the expected parameter space of our sample of stars with the iSpec, Turbospectrum, and MARCS.GES models. The $T_{\text {eff }}$ values of the grid are 4200, 4600, 5000, 5400, 5700, 6000, $6400 \mathrm{~K}$, so the grid has a slightly smaller step near the solar

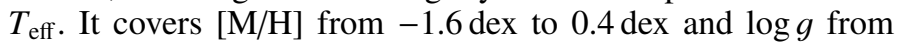
4.2 dex to 5.4 dex (both in steps of $0.4 \mathrm{dex}$ ). We know that some of our stars are in the subgiant branch and the $\log g$ values could be lower in those cases in order to more accurately match their spectra. The normalization grid properly normalizes areas of the spectra with large absorption lines. The smoothing after the normalization erases information from the weaker features, making it possible to use the grid to normalize spectra that do not exactly match the same parameters but that fall relatively close. Since the characterization of these subgiants is not the main focus of the work, we limited the parameter space of our normalizations. The most similar synthetic spectrum to each observed star, taking all three parameters into account, is selected as its reference spectrum. The parameters used for the selection are from ASPCAP or from literature sources when available (PASTEL, CPS, or HARPS-GTO).

The normalization is done by dividing the observed spectrum by its reference spectrum. The divided spectrum is smoothed afterward, taking the median value within $5.0 \mathrm{~nm}$ for each point in the spectrum. Dividing the observed spectra by this shape-fit results in a normalized spectrum. Our normalization method preserves the wide absorption features in the normalized spectrum, such as the hydrogen Brackett series lines $(1736.69 \mathrm{~nm}, 1681.11 \mathrm{~nm}, 1641.17 \mathrm{~nm}, 1611.37 \mathrm{~nm})$, that the default APOGEE normalization misses. The lines around $1681.11 \mathrm{~nm}$ and $1641.17 \mathrm{~nm}$, however, were removed from the line mask as synthesizing them with the necessary accuracy for parameter determination proved to be impossible.

\subsection{Line list}

The line list used is a compilation of lines from two different sources: The Vienna Atomic Line Database (VALD, Piskunov et al. 1995) and the APOGEE line list (Shetrone et al. $2015)^{4}$. They contain all of the relevant elemental lines as well as the molecular lines for $\mathrm{CO}, \mathrm{OH}, \mathrm{C}_{2}, \mathrm{CN}, \mathrm{CH}$, and $\mathrm{FeH}$. The solar spectrum presented in Wallace et al. (1996) was used to validate the final line list. Two synthetic spectra were created for the Sun, one with the VALD line list and another with the APOGEE line list. All the spectra, synthetic and solar, were convolved to the APOGEE-N resolution. Then individual $0.2 \mathrm{~nm}$ regions in each synthetic spectrum were compared to the spectrum from the Sun to inspect which line list provided the best fit for each region. Our line list was then composed by the best-fitting lines to the solar spectrum from the VALD and APOGEE line lists and contains 85334 lines.

\subsection{Line masks}

iSpec can use line masks to select the spectral features that will be considered for the determination of the atmospheric parameters. To create a list of line masks covering the best synthetic absorption lines, we compared a synthetic spectrum $\left(T_{\text {eff }} 5777 \mathrm{~K}\right.$, $[\mathrm{M} / \mathrm{H}] 0.0$ and $\log g=4.44 \mathrm{dex})$ to the solar spectrum by

\footnotetext{
4 The strength $(\log (g f))$ of the lines in this list was adjusted to better match a solar and an Arcturus synthetic spectrum. We did not change their values, which might have slightly affected our results.
} 
Wallace et al. (1996), both at APOGEE's resolution. The lines were selected by excluding the regions with lines that did not appear in the synthetic spectrum and the regions in the synthetic spectrum that did not match the solar spectrum as expected.

The inspection of each region in the synthesized and observed spectrum was made visually (see Fig. 7). The same line mask was used in all stars in our sample.

\section{6. $\chi^{2}$ fit and error estimation}

The mpfit.py code, based on MPFIT (Markwardt 2009), is used by iSpec to minimize the $\chi^{2}$ difference between the synthetic and the observed spectra. It uses a Levenberg-Marquardt leastsquares minimization to obtain the best fit to a given spectrum considering only the regions that are included in the line mask described above. All the errors cited for the stellar parameters in this work were calculated by iSpec from this code, and correspond to the formal $1 \sigma$ errors of each parameter, computed from the covariance matrix. The error calculation assumes uncertainties in the observed spectrum equal to the flux divided by the $\mathrm{S} / \mathrm{N}$ values published by ASPCAP. They are considered internal errors and are reported in each parameter for all stars in Table C.1.

To address how well our method is able to recover the parameters of each star under slightly different conditions, we added random Gaussian noise based on the flux errors to the normalized spectrum for each star. The Gaussian noise added had a zero mean and a standard deviation equal to the estimated flux error for each individual pixel. To avoid individual points or areas with large reported flux errors $(>1 / 10$ of the pixel flux value) that could skew the analysis, we restricted the value of Gaussian noise standard deviation to $1 / 10$ of the flux for those pixels.

We chose four stars with different spectral derived parameters from the CPS catalog and different S/N on their APOGEE spectrum and added to each spectrum the random Gaussian noise described above 100 times for each star. The derived parameters by iSpec from the 100 random Gaussian noise-added spectra are shown in Figs. 5 and 6 . These figures also show the strong degeneracies between parameters, as increases in derived $T_{\text {eff }}$ correspond to increases in $\log g$ and $[\mathrm{M} / \mathrm{H}]$ as well. Table 1 shows the mean values and standard deviation derived from the 100 iterations for each star. The consistency of our method across multiple syntheses can be confirmed, being able to recover consistent values for all the stars. As expected, the stars with the lowest S/N, 2M19172334+4412307 and 2M19040872+4936522, exhibit the largest differences between syntheses, reaching up to $\Delta T_{\text {eff }} \sim 100 \mathrm{~K}$ and $\Delta \log g=0.11 \mathrm{dex}$.

\subsection{Free and fixed parameters within iSpec}

iSpec allows the customization of the free parameters in each analysis during the $\chi^{2}$ fit and error minimization processes. All syntheses done for this work had the same group of free parameters: effective temperature $\left(T_{\text {eff }}\right)$, surface gravity $(\log g)$, metallicity $([\mathrm{M} / \mathrm{H}])$, micro-turbulent velocity $\left(v_{\text {mic }}\right)$, projected rotational velocity $(v \sin i)$, and spectral resolution. The input values were kept at $5800 \mathrm{~K}$ for the $T_{\text {eff }}, 0.0$ dex for the $[\mathrm{M} / \mathrm{H}]$, $4.5 \mathrm{dex}$ for the $\log g, 1.06 \mathrm{~km} \mathrm{~s}^{-1}$ for the $v_{\text {mic }}, 1.6 \mathrm{~km} \mathrm{~s}^{-1}$ for $v \sin i$, and 22000 for the resolution. The macro-turbulent velocity $\left(v_{\text {mac }}\right)$ follows an empirical relation provided by iSpec and built by the Gaia-ESO Survey based on their dataset and the $T_{\text {eff }}$, $\log g$, and $[\mathrm{M} / \mathrm{H}]$ of the stars. The limb darkening is a required parameter for syntheses using iSpec. We tested different input

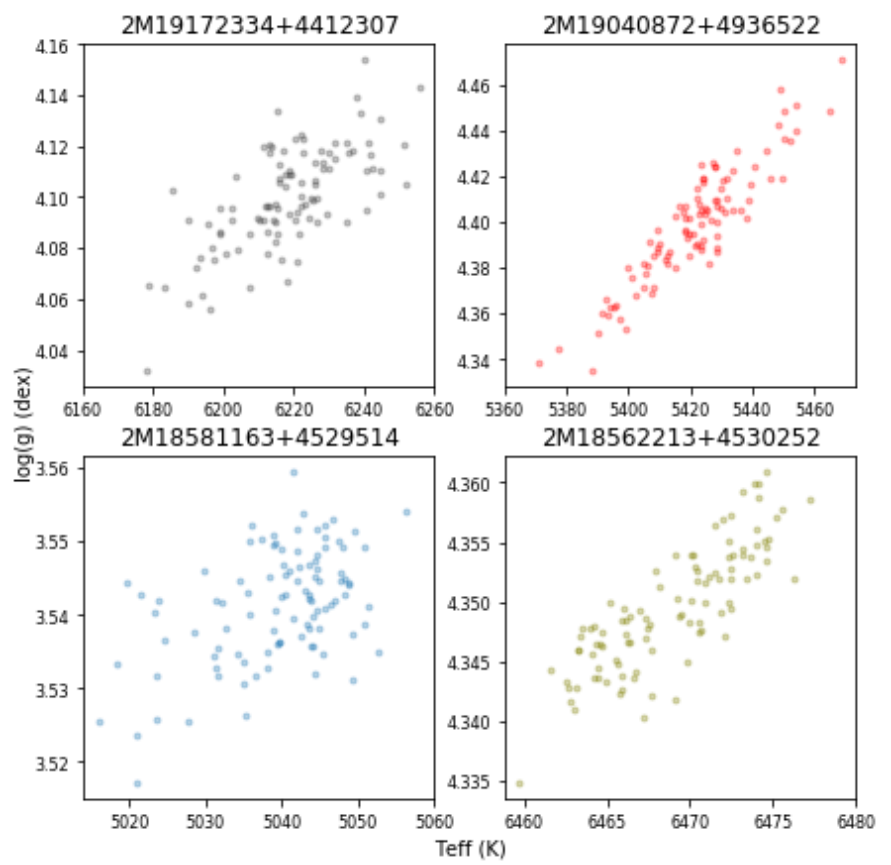

Fig. 5. Comparison between output parameters $\left(\log g\right.$ and $\left.T_{\text {eff }}\right)$ of four selected stars in 100 different iterations. Each point in the spectra had random Gaussian noise added to it.

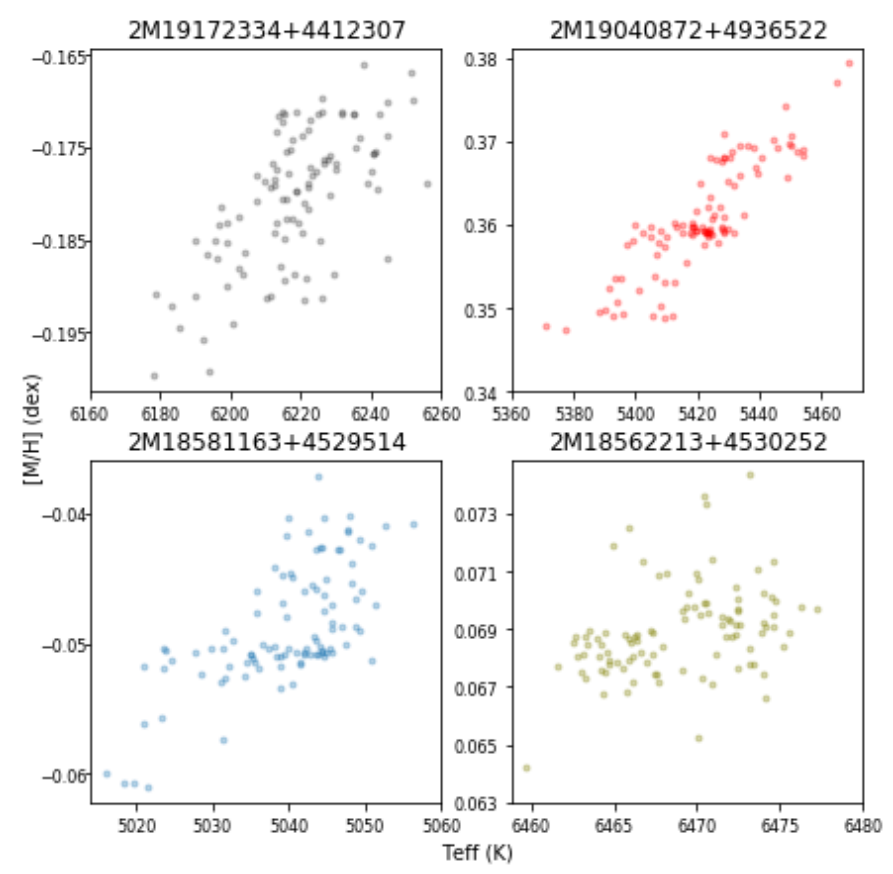

Fig. 6. Comparison between output parameters $\left([\mathrm{M} / \mathrm{H}]\right.$ and $\left.T_{\text {eff }}\right)$ of four selected stars in 100 different iterations. Each point in the spectra had random Gaussian noise added to it.

values compatible with solar-type stars, and since the output results have a negligible variation we decided to fix it at 0.6.

\section{Results}

\subsection{Solar spectra}

Although it was previously used to select lines for our line list, we retrieved the solar spectrum using our method to confirm its reliability. Since the Sun is the closest and best-characterized star 
Table 1. Parameters derived for the four stars selected.

\begin{tabular}{lccccccc}
\hline \hline APOGEE_ID & $S / N$ & $\overline{T_{\text {eff }} \pm \sigma_{T_{\text {eff }}}}$ & $T_{\text {eff }}^{C}$ & $\overline{\log g} \pm \sigma_{\log g}$ & $\log g^{C}$ & $\overline{[\mathrm{M} / \mathrm{H}]} \pm \sigma_{[\mathrm{M} / \mathrm{H}]}$ & {$[\mathrm{M} / \mathrm{H}]^{C}$} \\
\hline 2M19172334+4412307 & 121 & $6218 \pm 16 \mathrm{~K}$ & $6221 \mathrm{~K}$ & $4.10 \pm 0.02 \mathrm{dex}$ & $4.13 \mathrm{dex}$ & $-0.18 \pm 0.01 \mathrm{dex}$ & $-0.14 \mathrm{dex}$ \\
2M19040872+4936522 & 132 & $5421 \pm 18 \mathrm{~K}$ & $5487 \mathrm{~K}$ & $4.40 \pm 0.03 \mathrm{dex}$ & $4.31 \mathrm{dex}$ & $0.36 \pm 0.01 \mathrm{dex}$ & $0.34 \mathrm{dex}$ \\
2M18581163+4529514 & 216 & $5039 \pm 9 \mathrm{~K}$ & $4989 \mathrm{~K}$ & $3.54 \pm 0.01 \mathrm{dex}$ & $3.37 \mathrm{dex}$ & $-0.05 \pm 0.01 \mathrm{dex}$ & $-0.04 \mathrm{dex}$ \\
2M18562213+4530252 & 1095 & $6468 \pm 6 \mathrm{~K}$ & $6378 \mathrm{~K}$ & $4.35 \pm 0.01 \mathrm{dex}$ & $4.2 \mathrm{dex}$ & $0.07 \pm 0.01 \mathrm{dex}$ & $0.18 \mathrm{dex}$ \\
\hline
\end{tabular}

Notes. The $C$ indicates the literature (CPS) parameter for that star, and $\overline{\text { parameter }}$ and $\sigma$ indicate the average value and the standard deviation measured across all 100 iterations, respectively.

Table 2. Solar parameters derived from the Wallace et al. (1996) solar spectrum, and average results across 100 iterations with injected errors.

\begin{tabular}{lccc}
\hline \hline Parameter & Output & Average 100 iter. & $\sigma$ \\
\hline$T_{\text {eff }}(\mathrm{K})$ & 5764 & 5763 & 45 \\
$\log g($ dex $)$ & 4.49 & 4.51 & 0.09 \\
{$[\mathrm{M} / \mathrm{H}]($ dex $)$} & -0.04 & -0.02 & 0.03 \\
\hline
\end{tabular}

available, any reliable synthesis-based method should recover its spectral characteristics. Therefore, to calibrate and test iSpec with Turbospectrum in the $H$ band, a solar spectrum was synthesized using the methodology described in Sect. 3. We used the solar spectrum by Wallace et al. (1996) observed with the Fourier transform spectrometer at the Math-Pierce solar telescope on Kitt Peak, at a resolution of 300000 and high S/N. The spectrum was degraded down to $R=22500$ to match APOGEE's resolution. It was normalized using the template method described in Sect. 3.3 with a reference spectrum of $T_{\text {eff }}=5700 \mathrm{~K},[\mathrm{M} / \mathrm{H}]=0.0 \mathrm{dex}$, and $\log g=4.5 \mathrm{dex}$.

The observed and synthetic solar spectra are shown in Fig. 7. We took as the accepted solar parameters $T_{\text {eff }}=5777 \mathrm{~K}, \log g=$ $4.44 \mathrm{dex}$, and $[\mathrm{M} / \mathrm{H}]=0.0 \mathrm{dex}^{5}$. The synthesis done with our methodology provides a synthetic spectrum that matches the solar spectrum and derives just slightly lower values for the solar spectrum at APOGEE's resolution: $T_{\text {eff }}=5764 \pm 35 \mathrm{~K}$, $\log g=4.49 \pm 0.07 \mathrm{dex},[\mathrm{M} / \mathrm{H}]=-0.04 \pm 0.02$ dex. Because the Wallace et al. (1996) solar spectrum did not provide flux errors, we estimated errors on the parameters from 100 syntheses of the solar spectrum with injected Gaussian noise of 1/1000 of the flux at each pixel of the spectrum (similar to the process described in Sect. 3.6). The average values for $T_{\text {eff }}, \log g$, and $[\mathrm{M} / \mathrm{H}]$ from the 100 syntheses are shown in Table 2 . As expected, the parameters obtained in each synthesis are not independent of each other, and there are degeneracies between them, as shown by the correlation between $T_{\text {eff }}$ and $[\mathrm{M} / \mathrm{H}]$ in Fig. 8. Within the 100 syntheses, 87 have estimated $T_{\text {eff }}$ values within $5777 \pm 50 \mathrm{~K}$, and 65 of them have $[\mathrm{M} / \mathrm{H}]$ within $0.0 \pm 0.02 \mathrm{dex}$. These results also show standard deviations of $45 \mathrm{~K}$ for $T_{\text {eff }}, 0.09$ dex for $\log g$, and $0.03 \mathrm{dex}$ for $[\mathrm{M} / \mathrm{H}]$. The parameter analysis indicates a high degree of consistency between the iterations of our pipeline. However, in some cases, the retrieved parameters diverge significantly from the expected values. In particular, we registered unusual differences in $T_{\text {eff }}$ of $-150 \mathrm{~K}$ and -0.10 dex for $[\mathrm{M} / \mathrm{H}]$.

\subsection{APOGEE FGK sample}

The 3748 stars in our sample were synthesized using the methodology described in Sect. 3. Figure 9 shows the distribution

\footnotetext{
5 https://nssdc.gsfc.nasa.gov/planetary/factsheet/ sunfact.html
}

of $\log g$ versus $T_{\text {eff }}$ values for our sample stars, with overplotted isochrones from PARSEC (Bressan et al. 2012) made with $[\mathrm{M} / \mathrm{H}]=0.0$ dex. As expected from our selection of objects described in Sect. 2, the large majority of the stars synthesized fall within a small range of parameters, corresponding to FGK main-sequence stars. They also mostly fall within the range of the isochrones, giving us more confidence on the values. A small number of stars exhibit temperatures lower than $5000 \mathrm{~K}$. These stars also appear as subgiants in the CPS sample $(\log g<3.9$ dex) and as cooler dwarfs in the PASTEL catalog. The $\log g$ for these colder stars seems to be slightly underestimated as they are outside the range of the isochrones. Moreover, the $\log g$ for some of the hottest stars in the sample seems to be overestimated. A similar behavior for $\log g$ values of FGK dwarfs in the HR diagram has been observed after using the ionization balance method to derive $\log g$. Therefore, we decided to apply the corrections based on trigonometric $\log g$ derived in Delgado Mena et al. (2017; see Eqs. (1)-(3) in that paper) to $\log g$ values above $3.75 \mathrm{dex}$. Our corrected $\log g$ values are plotted in gray in Fig. 9, and are included as $\log g_{\text {cor }}$ in Table C.1.

The $[\mathrm{M} / \mathrm{H}]$ histograms for each significant subsample are shown in Fig. 10. The $[\mathrm{M} / \mathrm{H}]$ values by iSpec follow a Gaussian distribution that peaks around $0.0 \mathrm{dex}$, which is the Sun's metallicity. This was expected for our APOGEEonly subsample, since we restricted the sample by metallicity $(-0.5<[\mathrm{M} / \mathrm{H}]<+0.5$ dex only $)$. Analysis of metallicities in the solar neighborhood sample (as seen, for example, in Sousa et al. 2008) show a slightly lower average value of $[\mathrm{Fe} / \mathrm{H}]=-0.09$.

To reveal the challenges of synthesizing APOGEE spectra, both the observed APOGEE and synthesized spectra for an example star is shown in Fig. $11^{6}$. This star was chosen as a representative of the sample and of the output produced by the method, both the synthetic spectrum generated and the parameters derived by the pipeline. For the example star, the APOGEE spectrum is shown in black, the best-fit spectrum (synthetic spectrum created with the parameters found by our methodology) is shown in red, and the synthetic spectrum created with the full line list and using the literature parameters is shown in blue. Therefore, the blue spectrum represents the expected spectrum for the example star considering its derived parameters from high-resolution optical spectra.

The star 2M19144528+4109042 (KOI-85, Fig. 11) has three substellar companions detected (Borucki et al. 2011; Rowe et al. 2014). Our spectroscopic values are higher than the optical values from the CPS catalog, except for the metallicity, which is slighter lower. However, considering errors, the our values and those from the CPS catalogs are consistent. The two synthetic spectra are similar between themselves and are a match to the observed spectrum; the most significant differences appear around the strong hydrogen line in the $1681.1 \mathrm{~nm}$ region and

\footnotetext{
6 Comparison spectra for 3 additional stars is available in the appendix.
} 
P. Sarmento et al.: Derivation of parameters for 3748 FGK stars using $H$-band spectra from APOGEE Data Release 14

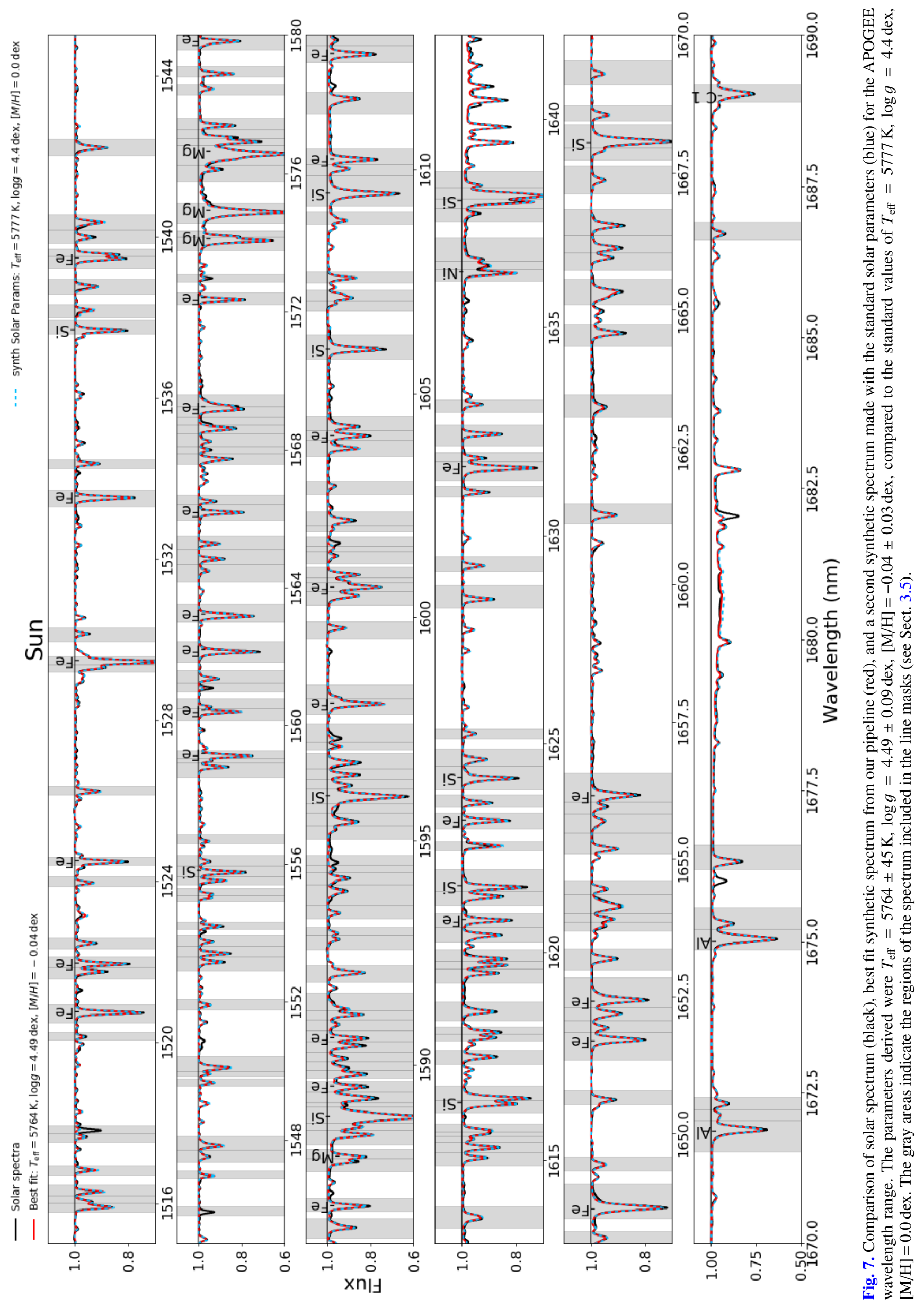




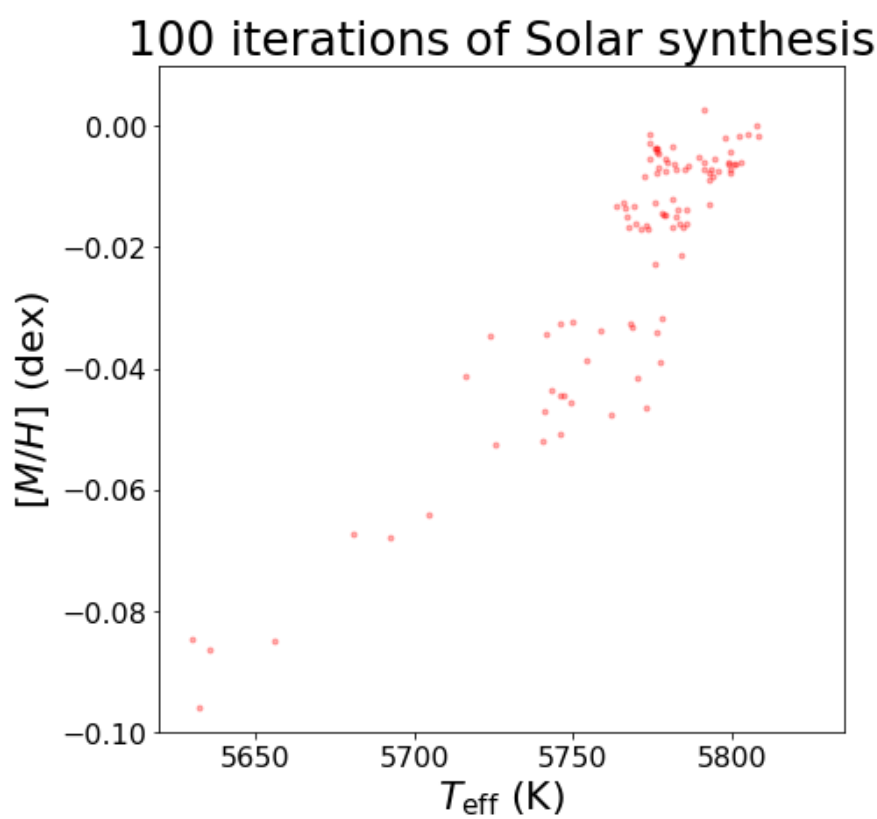

Fig. 8. Output $T_{\text {eff }}$ and $[\mathrm{M} / \mathrm{H}]$ across 100 iterations of our pipeline with the solar spectrum.

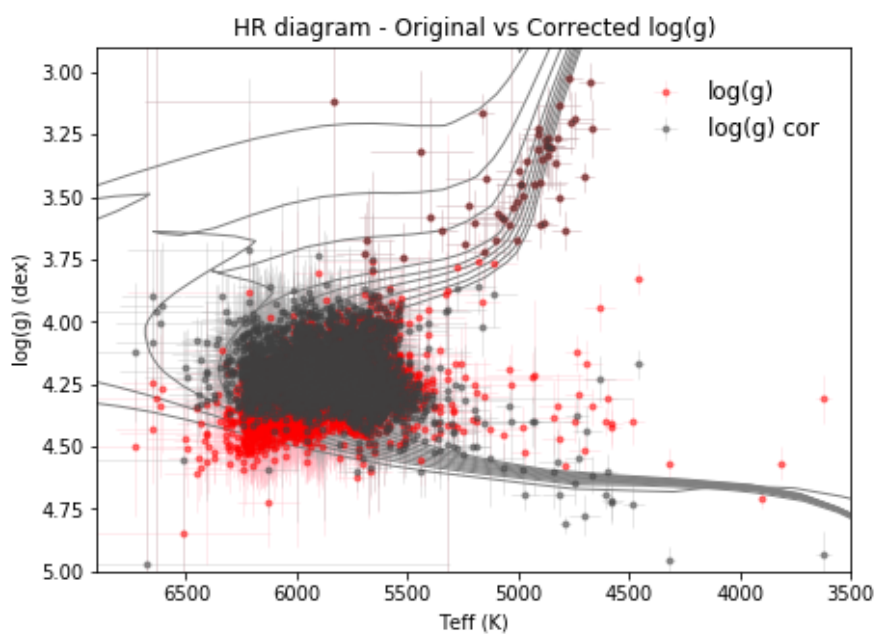

Fig. 9. Pseudo-HR diagram of our results with the full sample, plotting both the derived $\log g$ and $\log g$ after corrections based on trigonometric $\log g$ from Delgado Mena et al. (2017) against $T_{\text {eff }}$ for all the stars in the sample. Worst outlier stars are excluded from this plot. PARSEC isochrones with $[\mathrm{M} / \mathrm{H}]=0.0$ dex from Bressan et al. (2012) are overplotted.

continuum around $1640 \mathrm{~nm}$. There are also differences in line depths across the spectrum, most noticeably the Si line at wavelength $1596 \mathrm{~nm}$. This can be explained by the differences in metallicity between the two synthetic spectra.

We realize that comparing the best-fit synthetic spectra with those created using literature parameters is not a perfect comparison because of the inhomogeneities: values derived with different spectra, methods, models, and normalizations. Using optical spectra to derive parameters for our sample stars and using them to generate NIR spectra could result in a better comparison for our method. However, different line lists and masks would still be required for the derivation of parameters with optical wavelength spectra, which can result in differences when comparing

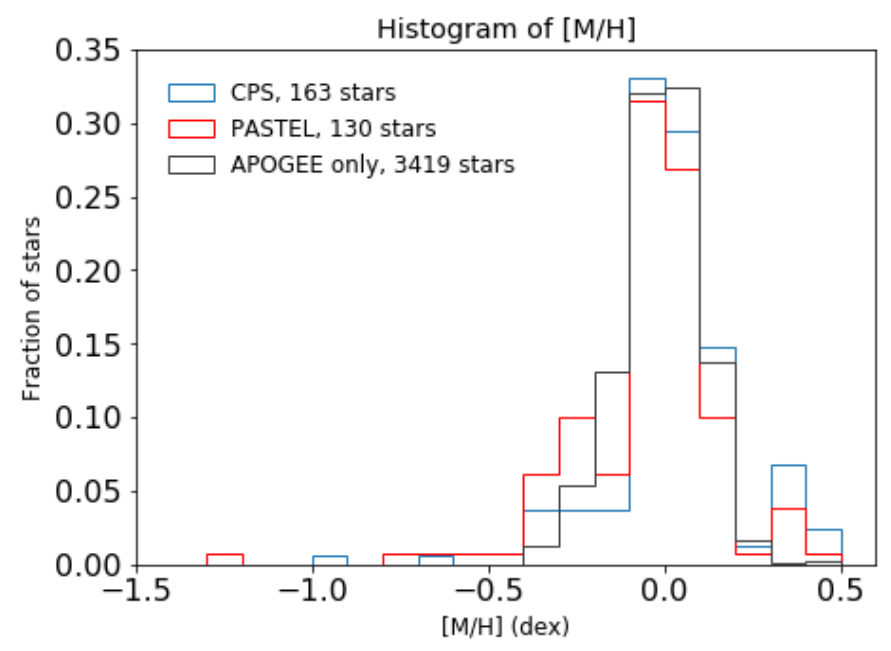

Fig. 10. Histogram with the metallicity distribution of the final sample, divided by subsamples of stars in common with other catalogs. Values plotted are the output of our pipeline. Worst outlier stars are excluded from this histogram.

them with $H$-band results. The comparisons present in this paper are therefore a compromise between these issues.

We are also aware that the visual comparisons between the spectra presented in this paper are difficult to judge accurately, as the minimization process takes into account flux errors that are not shown here. The spectra shown in Fig. 11 is intended more as a visual display of the method's results than its scientific precision.

\section{Discussion}

\subsection{ASPCAP comparison}

In this subsection, we compare our results to the derived ASPCAP parameters for our sample of stars. ASPCAP was developed to derive spectroscopic parameters for the APOGEE survey, and its parameter determination pipeline is optimized for giant stars. As mentioned in Sect. 2, $[\mathrm{M} / \mathrm{H}], T_{\text {eff }}$, and $\log g$ are provided for all stars in the sample. ASPCAP values for $[\mathrm{M} / \mathrm{H}]$ and $T_{\text {eff }}$ for all stars are calibrated using independent methods, but the $\log g$ values for dwarf stars are not independently calibrated and differ from isochrone values. We decided to compare our values for $T_{\text {eff }}$ and $[\mathrm{M} / \mathrm{H}]$ with the calibrated values provided by ASPCAP for our sample of stars. We also compare our raw values for $\log g$ with the available ASPCAP $\log g$ values.

Figure 12 presents the $T_{\text {eff }}$ comparison, while the $[\mathrm{M} / \mathrm{H}]$ comparison is presented in Fig. 13. These comparisons include both the subsamples characterized in optical wavelengths and the 3419 stars from which only ASPCAP parameters are available. Although only the ASPCAP values are compared, we distinguish the subsamples characterized in the optical by color (CPS - blue; PASTEL - red; HARPS-GTO - yellow), while the stars in black have only been characterized by ASPCAP and our pipeline. This is done for reasons of visual clarity and consistency.

In Table 3 the average differences between our output and the ASPCAP values are presented. Given the large sample of stars (3419) compared to our subsample observed in the optical, the measured differences between our pipeline's values and ASPCAP's parameters are within one standard deviation, which means that our method can provide consistent parameters for solar-type stars in the NIR. 
P. Sarmento et al.: Derivation of parameters for 3748 FGK stars using $H$-band spectra from APOGEE Data Release 14

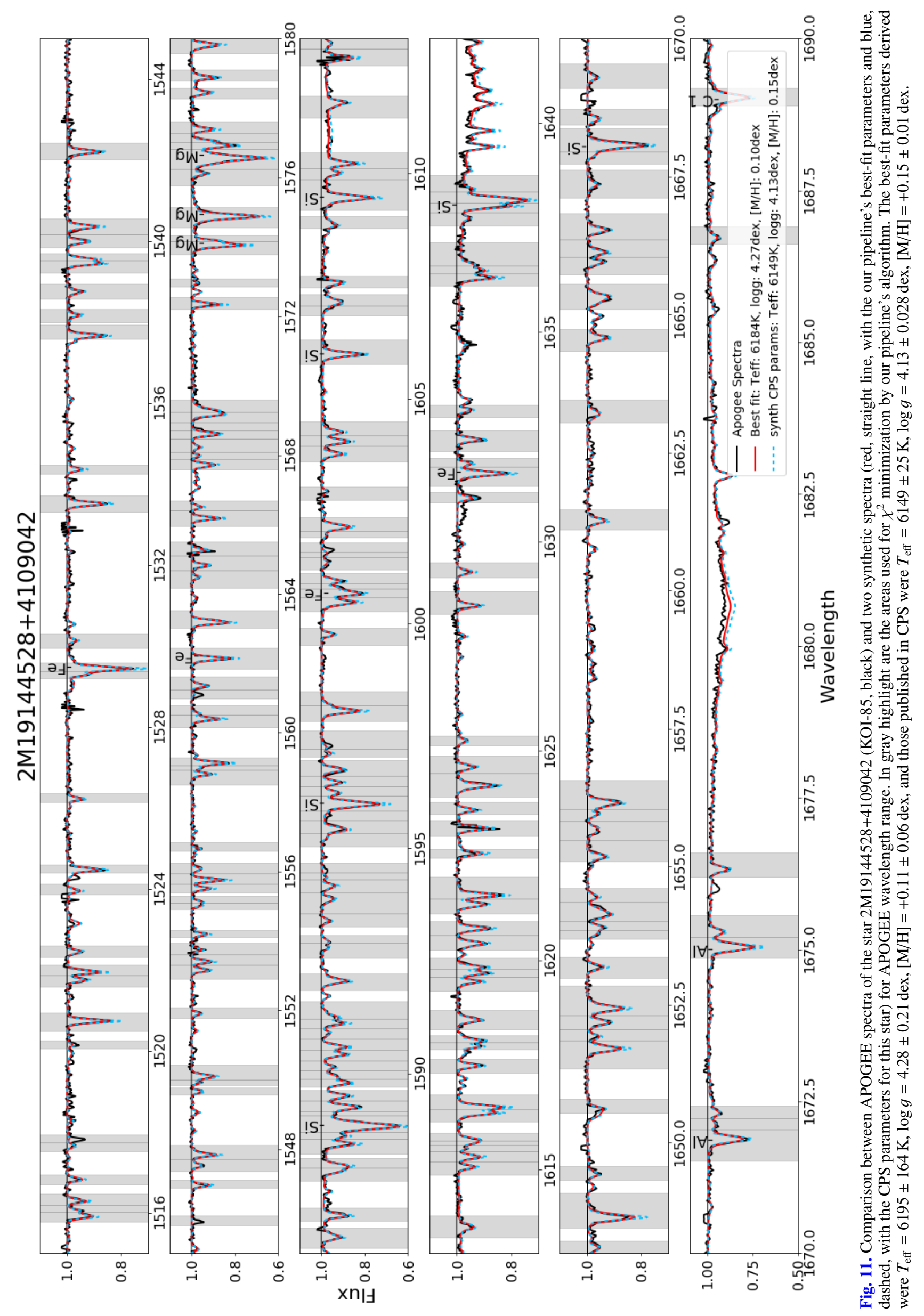




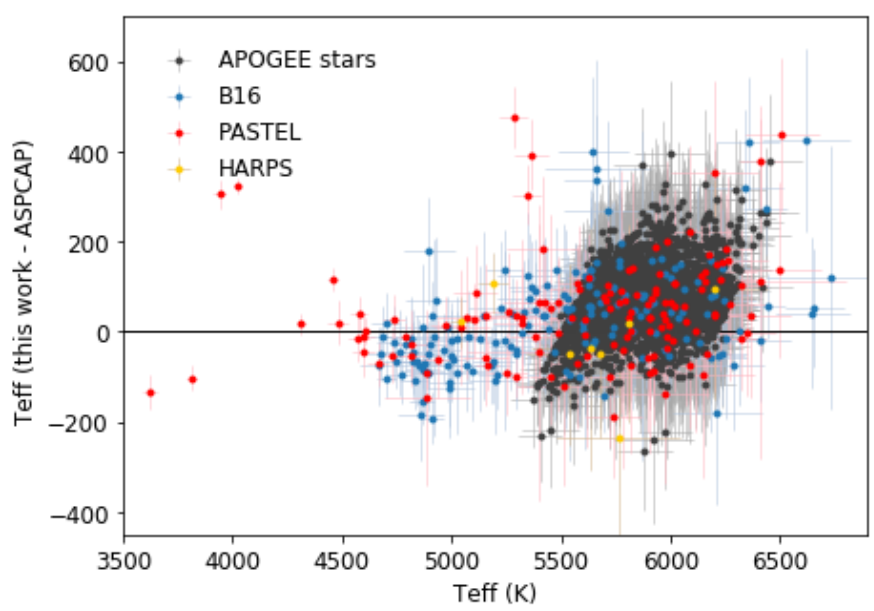

Fig. 12. Diagram representing the output $T_{\text {eff }}$ from our pipeline, and the difference between it and the values published by ASPCAP for each star in our sample. Only ASPCAP values are compared, and the different colors are used to distinguish between stellar subsamples. Errors displayed on both axes are our pipeline's estimates using the covariance matrix.

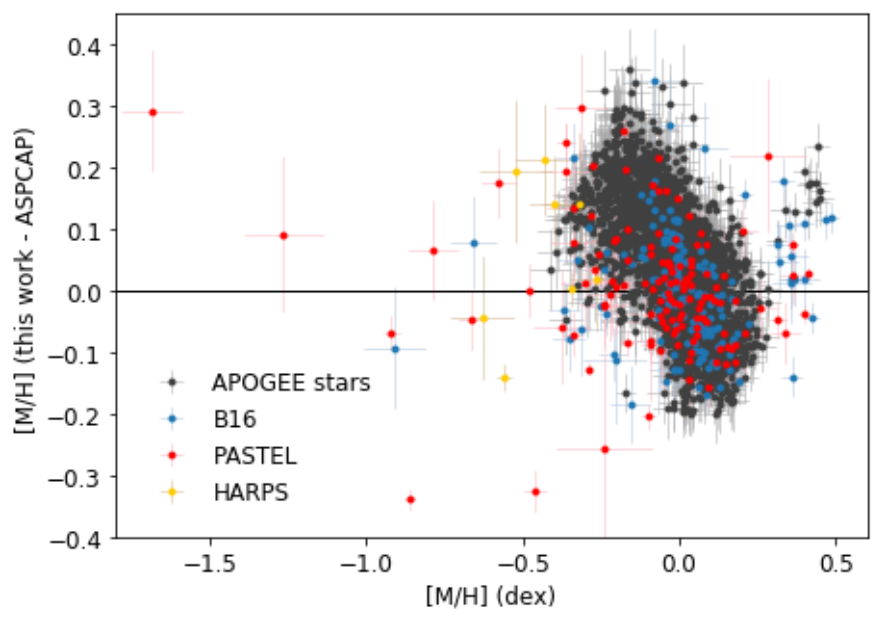

Fig. 13. Diagram representing the output $[\mathrm{M} / \mathrm{H}]$ from our pipeline, and the difference between it and the values published by ASPCAP for each star in our sample. Only ASPCAP values are compared, and the different colors are used to distinguish between stellar subsamples. Errors displayed on both axes are our pipeline's estimates using the covariance matrix.

The median estimated $T_{\text {eff }}$ is $62 \mathrm{~K}$ above ASPCAP values, although the difference varies between $-150 \mathrm{~K}$ and $+300 \mathrm{~K}$. The fact that the median difference is still within the margin of error for both pipelines is an encouragement for the accuracy of our method.

A small difference is present in metallicity as well, which can clearly be seen in Fig. 13 and in Table 3. We find an average difference between our values for $[\mathrm{M} / \mathrm{H}]$ and those in ASPCAP of +0.04 dex. Compared with the differences registered against parameters measured in the optical, we find this value to be above the median differences for the CPS values $(-0.02$ dex $)$ and at the same level as the PASTEL values $(+0.04$ dex, see Sect. 5.2). It is still below the HARPS-GTO difference of +0.16 dex. Additionally, there seems to be a trend, observable in Fig. 13, that results in lower $\Delta[\mathrm{M} / \mathrm{H}]$ values for stars with higher $[\mathrm{M} / \mathrm{H}]$, with a small number of high $[\mathrm{M} / \mathrm{H}]$ outliers. A larger sample size of both low- and high- $[\mathrm{M} / \mathrm{H}]$
Table 3. Median differences for ASPCAP parameters.

\begin{tabular}{lcc}
\hline \hline Parameter & Stars & Median (this work - ASPCAP) \\
\hline$T_{\text {eff }}$ & 3670 & $+62 \mathrm{~K}$ \\
{$[\mathrm{M} / \mathrm{H}]$} & 3668 & $+0.04 \mathrm{dex}$ \\
$\log g$ & 3622 & $-0.01 \mathrm{dex}$ \\
\hline
\end{tabular}

Notes. The "Stars" column represents the number of stars with this ASPCAP parameter between the two original samples.

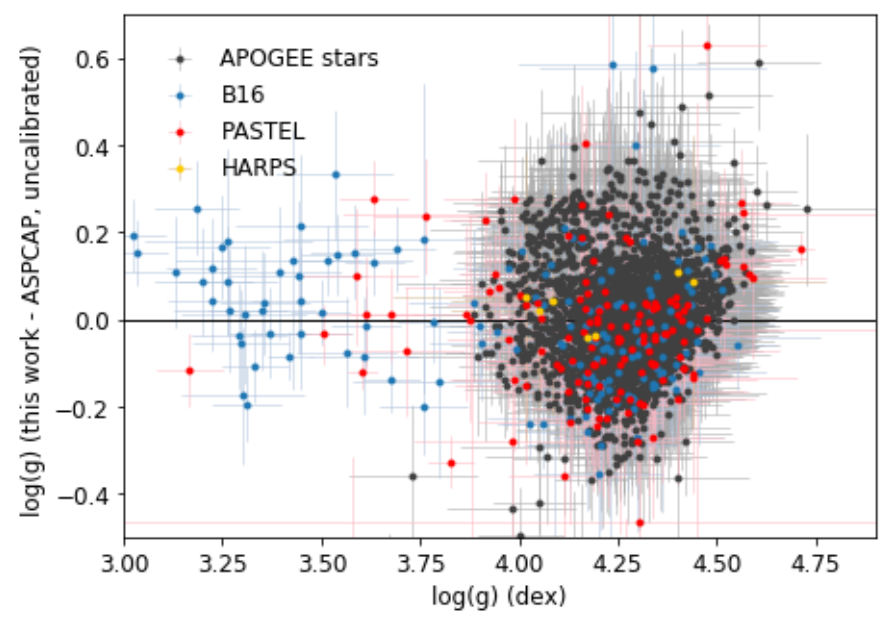

Fig. 14. Diagram representing the uncorrected output $\log g$ from our pipeline (see Fig. 9), and the difference between it and the values published by ASPCAP for each star in our sample. Errors displayed on both axes are our pipeline's estimates using the covariance matrix.

stars seems necessary to better characterize the behavior of the pipeline for that extended parameter space.

We display a comparison between published ASPCAP $\log g$ values and our raw output $\log g$ in Fig. 14. The plot shows that the distributions of our $\log g$ values and the ASPCAP values are quite similar, with a median difference of -0.007 dex. The difference between the two $\log g$ values is also around or below 0.4 dex for most stars in the sample, showing that the values obtained with our method are actually consistent with the available ASPCAP values. However, several studies comparing asteroseismic and spectroscopic $\log g$ have demonstrated that spectroscopic values need to be calibrated (e.g., Holtzman et al. 2015; Mortier et al. 2014a). In addition, the comparison with stellar isochrones points to the inaccuracy of spectroscopic $\log g$ values. Therefore, we decided to apply the correction as shown in Fig. 9 and explained in Sect. 4.2.

\subsection{Other literature}

The derived parameters for the stars in common with optical surveys are plotted in Figs. 15-17, and are listed in Table C.1. The calculated differences between our method's parameters and those published in CPS, PASTEL, and HARPS-GTO are presented in Tables 4-6. For HARPS-GTO, and since there are only eight stars in the sample, each individual result is included.

The spectra displayed in Fig. 11 show that our pipeline can minimize $\chi^{2}$ and match the APOGEE observed spectrum with a synthesized spectrum to the required precision. Figure 15 shows our derived $T_{\text {eff }}$ for each of the three subsamples of stars plotted against the difference between it and literature parameters for the same stars. While the majority of the results have differences 


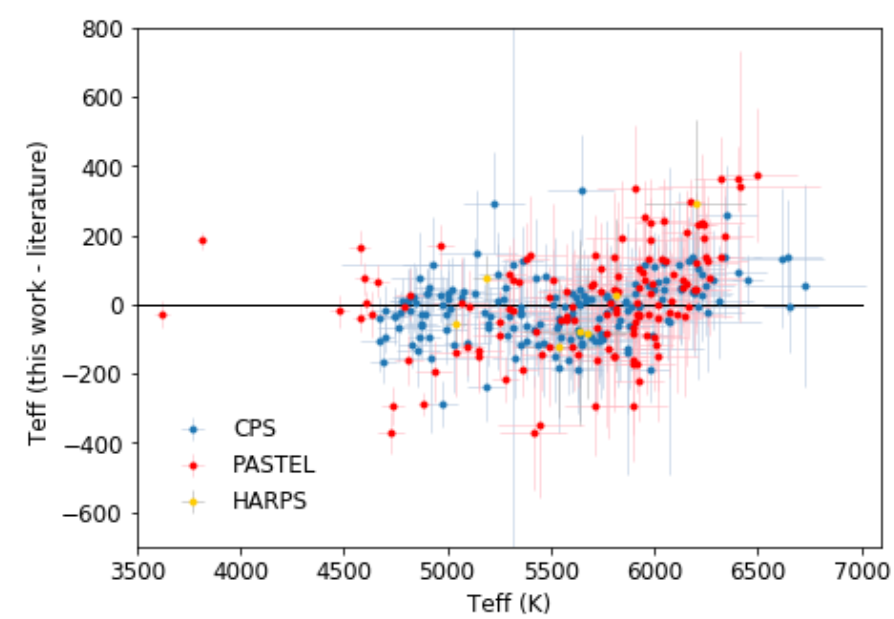

Fig. 15. Diagram plotting the output $T_{\text {eff }}$ of our pipeline against the difference between it and the values published by CPS, PASTEL, and the HARPS-GTO program. Errors included are our pipeline's estimates using the covariance matrix.

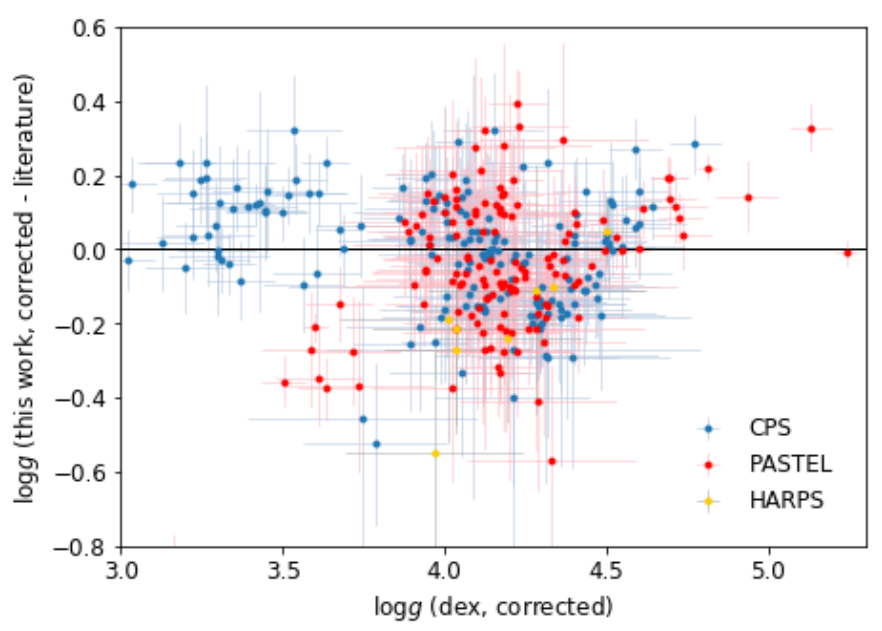

Fig. 16. Diagram plotting the output $\log g_{\text {cor }}$ of our pipeline against the difference between it and the values published by CPS, PASTEL, and the HARPS-GTO program. Errors included are our pipeline's estimates using the covariance matrix.

smaller than $200 \mathrm{~K}$, an upward trend is noticeable for stars above $6000 \mathrm{~K}$, resulting in an overestimation of temperatures for the hottest stars in the sample. Figure 16 shows our derived $\log g_{\text {cor }}$ for each of the three subsamples of stars plotted against the difference between it and literature parameters for the same stars. This plot shows that, apart from our small subsample of subgiant stars, the surface gravity of most of our sample stars falls within a small range of values (4.0 to $4.6 \mathrm{dex}$ ) and $\Delta \log g$ ranging from -0.4 to +0.4 for these stars. Forty-nine stars from the CPS sample and 31 from the PASTEL sample fall outside that range. For those stars, the $\Delta \log g$ values between our parameters and their literature values are different between samples, with our method resulting in values above the literature values for CPS stars and below for PASTEL. This difference can be explained by the different methods used to determine the parameters. Figure 17 shows our derived $[\mathrm{M} / \mathrm{H}]$ for each of the three subsamples of stars plotted against the difference between it and the literature parameters for the same stars. A small gap appears, with our method reporting only five stars with $[\mathrm{M} / \mathrm{H}]$ between +0.2 and +0.3 dex. This could be a statistical anomaly and due

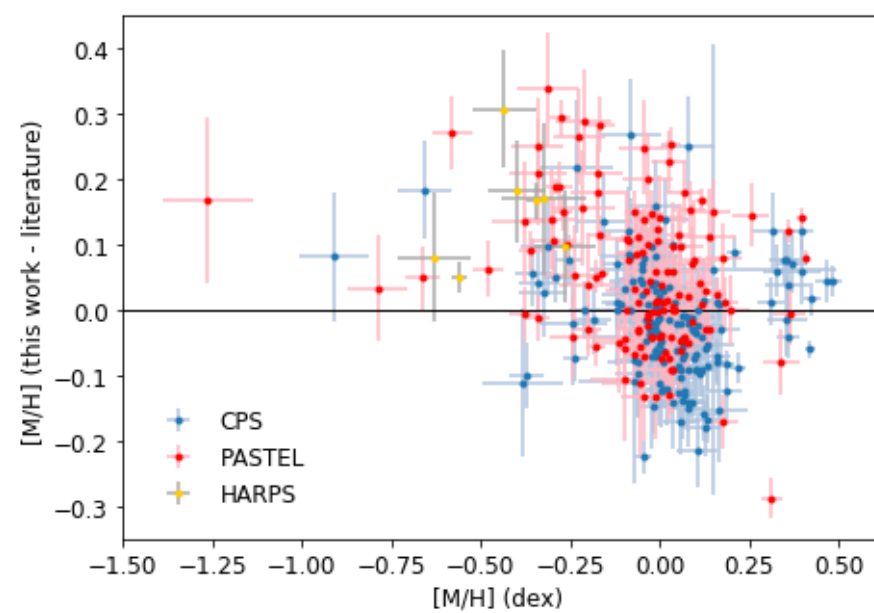

Fig. 17. Diagram plotting the output $[\mathrm{M} / \mathrm{H}]$ of our pipeline against the difference between it and the values published by CPS, PASTEL, and the HARPS-GTO program. Errors included are our pipeline's estimates using the covariance matrix.

Table 4. Average differences in derived parameters between our method and CPS parameters.

\begin{tabular}{lc}
\hline \hline Parameter & Median (pipeline - CPS) \\
\hline$T_{\text {eff }}$ & $-7 \mathrm{~K}$ \\
$\log g_{\text {cor }}$ & $+0.01 \mathrm{dex}$ \\
{$[\mathrm{M} / \mathrm{H}]$} & $-0.02 \mathrm{dex}$ \\
\hline
\end{tabular}

Notes. Sample size is 168 stars. The median is shown to decrease the effect of outliers. The $\log g_{\text {cor }}$ value compared is our calibrated pipeline's output.

Table 5. Average differences in derived parameters between our method and the PASTEL parameters.

\begin{tabular}{lc}
\hline \hline Parameter & Median (pipeline - PASTEL) \\
\hline$T_{\text {eff }}$ & $+7 \mathrm{~K}$ \\
$\log g_{\text {cor }}$ & $-0.06 \mathrm{dex}$ \\
{$[\mathrm{M} / \mathrm{H}]$} & $+0.04 \mathrm{dex}$ \\
\hline
\end{tabular}

Notes. Sample size is 153 stars. The median is shown to decrease the effect of outliers. The $\log g_{\text {cor }}$ value compared is our calibrated pipeline's output.

only to the number of stars in the sample. Figures 15-17 and Tables 4 and 5 show that the method does not seem to have systematic errors in any of the three synthesized parameters $\left(T_{\text {eff }}\right.$, $\log g$, and $[\mathrm{M} / \mathrm{H}])$ as the error margins for our parameter estimates are within the optical measurements.

In the case of the PASTEL stars, some of the discrepancies can be explained by the fact that PASTEL presents $[\mathrm{Fe} / \mathrm{H}]$ and not $[\mathrm{M} / \mathrm{H}]$, and that their values for the $T_{\text {eff }}$ come from different sources. This is not true for the CPS parameters, which are uniformly calculated and have better precision than PASTEL parameters.

The largest difference between our derived parameters and the literature values is found for the $[\mathrm{M} / \mathrm{H}]$ measurements in the HARPS-GTO sample (average $+0.16 \mathrm{dex}$ ). This difference may be caused by either the sample size, as the larger PASTEL and CPS samples do not show these differences, or just a systematic error caused by the method used, as the HARPS-GTO 
Table 6. Differences in derived parameters between our method and HARPS-GTO (our pipeline vs. the literature).

\begin{tabular}{lcccccc}
\hline \hline ID (2mass) & $T_{\text {eff }}(\mathrm{K})$ & $T_{\text {eff }}^{H}$ & $\log g_{\text {cor }}(\operatorname{dex})$ & $\log g^{H}(\mathrm{dex})$ & {$[\mathrm{M} / \mathrm{H}](\mathrm{dex})$} & {$[\mathrm{M} / \mathrm{H}]^{H}(\mathrm{dex})$} \\
\hline 2M03402202-0313005 & 5762 & 5884 & 3.96 & 4.52 & -0.52 & -0.82 \\
2M04042029-0439185 & 5192 & 5116 & 4.50 & 4.45 & -0.34 & -0.51 \\
2M07385132-0527558 & 5635 & 5716 & 4.01 & 4.20 & -0.32 & -0.49 \\
2M08172935-0359221 & 5678 & 5762 & 4.04 & 4.31 & -0.40 & -0.58 \\
2M15074648+0852472 & 5811 & 5782 & 4.04 & 4.25 & -0.43 & -0.74 \\
2M15124763-0109577 & 5043 & 5096 & 4.34 & 4.44 & -0.56 & -0.61 \\
2M16302844+0410411 & 6200 & 5908 & 4.28 & 4.39 & -0.63 & -0.71 \\
2M16410822-0251258 & 5542 & 5665 & 4.19 & 4.43 & -0.26 & -0.36 \\
\hline
\end{tabular}

Notes. The $H$ denotes the literature value for the parameter. The $\log g_{\text {cor }}$ value compared is our calibrated pipeline's output.

parameters were measured using the EW method. The fact that HARPS-GTO results are $[\mathrm{Fe} / \mathrm{H}]$ and not $[\mathrm{M} / \mathrm{H}]$ may also have contributed to this difference. All the HARPS-GTO stars in common with our sample are relatively metal poor, having $[\mathrm{M} / \mathrm{H}]$ ranging from -0.82 to -0.36 dex, which means they are in a relatively small parameter space and may not represent a real distribution of stars like the other studied samples.

Our parameter estimates resulted in a high number of stars within the PASTEL sample for which the difference with respect to the PASTEL values was significant, with 21 out of 153 stars having parameters that differ significantly from literature values $\left(|\Delta[\mathrm{M} / \mathrm{H}]|>0.35 \mathrm{dex} ;|\Delta \log g|>0.5 \mathrm{dex} ;\left|\Delta T_{\text {eff }}\right|>400 \mathrm{~K}\right)$. The worst of these outliers are either very metal-poor stars $([\mathrm{M} / \mathrm{H}]<-1.5 \mathrm{dex})$ or cold $\mathrm{K}$ and $\mathrm{M}$ dwarfs $\left(T_{\text {eff }}<4000 \mathrm{~K}\right)$. Even though our pipeline is not optimized for these stars, their existence must be taken into account when synthesizing large samples of stars.

An additional explanation for any discrepancies can be that APOGEE spectra are in the $H$ band (infrared) and CPS, PASTEL and HARPS-GTO results were calculated using spectra in the optical wavelength range.

\subsection{Further discussion}

As described in Sects. 5.1 and 5.2, in Tables 3 and 4, and in Figs. 13-15, comparisons between previous observations in the optical and in the NIR for our sample stars show that there are differences between our parameters and the ones in literature. This section proposes and analyzes some explanations for these differences.

Errors in the line list and/or the models used for the synthesis might be one reason for these discrepancies. The synthetic spectra match the observed one to a very high degree of accuracy (see Figs. 7 and 11), so it is unlikely this is the case. Tests with solar spectrum show that our pipeline can provide accurate parameters for a solar-type star with APOGEE resolution (see Sect. 4.1), so it is unlikely that this is the explanation for all discrepancies found between this method's parameters and the ones previously derived in the literature.

Another hypothesis is that there is a difference between $[\mathrm{M} / \mathrm{H}]$ and $[\mathrm{Fe} / \mathrm{H}]$ (iron abundances) for these stars, as the CPS value we are comparing is $[\mathrm{M} / \mathrm{H}]$ and both PASTEL and HARPS-GTO samples published [Fe/H] instead. Valenti \& Fischer (2005) found different distributions for these two parameters in nearby FGK stars, with $[\mathrm{M} / \mathrm{H}]$ having an average of $0.0 \mathrm{dex}$ and $[\mathrm{Fe} / \mathrm{H}]$ of +0.04 dex. It should be noted, however, that Haywood (2001) has found $[\mathrm{Fe} / \mathrm{H}]=0$ for stars in the solar neighborhood, and Rocha-Pinto \& Maciel (1996) measured an average of $[\mathrm{Fe} / \mathrm{H}]=-0.2$ dex for $\mathrm{G}$ dwarfs in the solar

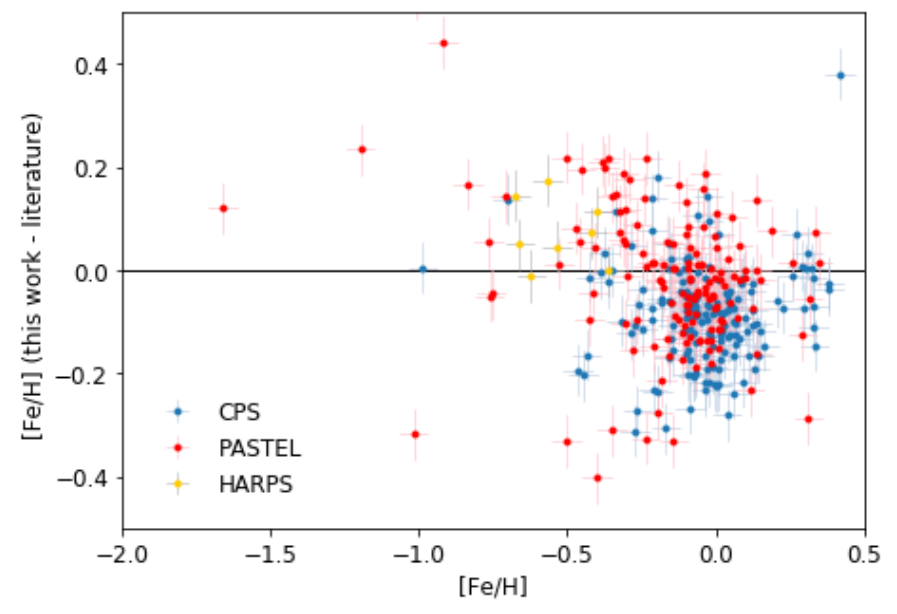

Fig. 18. Comparison between iron abundance derived by our pipeline and literature values.

Table 7. Average differences between $[\mathrm{Fe} / \mathrm{H}]$ derived with this method and literature values for $[\mathrm{Fe} / \mathrm{H}]$.

\begin{tabular}{lcc}
\hline \hline Sample & Stars & Median (pipeline - literature) \\
\hline CPS & 168 & $-0.08 \mathrm{dex}$ \\
PASTEL & 153 & $-0.02 \mathrm{dex}$ \\
HARPS-GTO & 8 & $+0.06 \mathrm{dex}$ \\
\hline
\end{tabular}

Notes. The median is shown to decrease the effect of outliers.

neighborhood. Therefore, comparing the overall metallicities for each star with their iron abundances can lead to unwanted disparities. We determined $[\mathrm{Fe} / \mathrm{H}]$ for each star in the sample using our pipeline, and compared them to values in the literature. These syntheses were done having only the Fe abundance as a free parameter and fixing the other parameters to the results previously derived by our pipeline. The abundances derived are summarized in Fig. 18 and are available in Table C.1 in the appendix.

In Table 7 it is still clear that the $[\mathrm{Fe} / \mathrm{H}]$ value given by our pipeline is very close to the literature values. The largest measured differences are found when comparing our values with CPS $(\Delta[\mathrm{Fe} / \mathrm{H}]=-0.08 \mathrm{dex})$, while the median difference in the PASTEL sample comparison is actually -0.02 dex. In the case of HARPS-GTO stars, our results are now in better agreement with theirs $(\Delta[\mathrm{Fe} / \mathrm{H}]=+0.06 \mathrm{dex})$ as we are comparing $[\mathrm{Fe} / \mathrm{H}]$ rather than $[\mathrm{M} / \mathrm{H}]$. These results show us that the method is robust and can provide not only $[\mathrm{M} / \mathrm{H}]$, but also $[\mathrm{Fe} / \mathrm{H}]$ for the analyzed stars. 

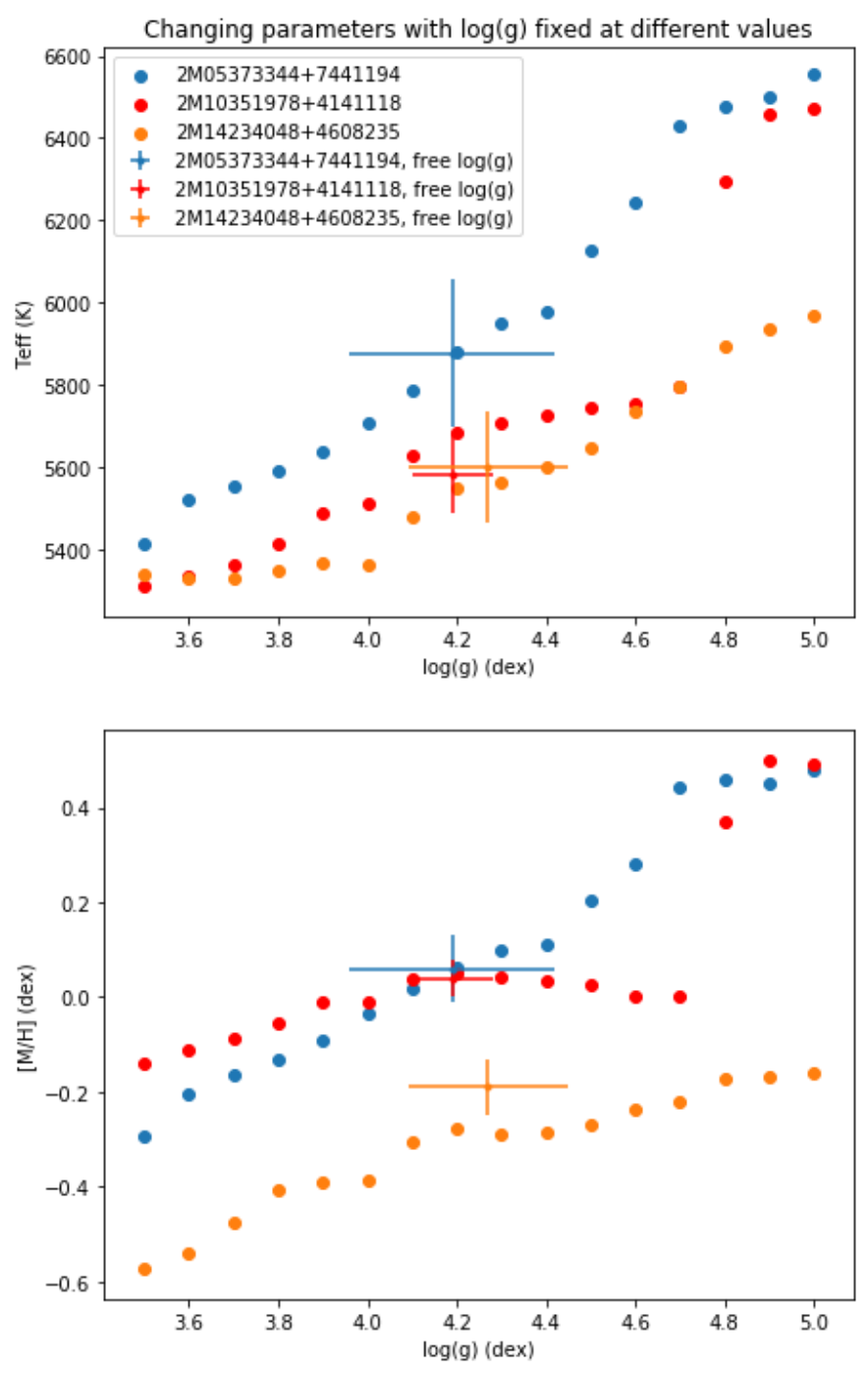

Fig. 19. Diagram representing the output parameters with fixed surface gravity at different values between $\log g=3.5 \mathrm{dex}$ and $\log g=5.0 \mathrm{dex}$. The data for three different stars is presented, 2M05373344+7441194 (blue), 2M14234048+4608235 (orange), and 2M10351978+4141118 (red), with the measured values for each indicated by an error bar of the same color.

The fact that the spectroscopic $\log g$ needs to be revised makes us wonder about the impact of an incorrect $\log g$ on the simultaneous derivation of $T_{\text {eff }}$ and $[\mathrm{M} / \mathrm{H}]$. Syntheses with lower values for $\log g$ can result in lower values for $T_{\text {eff }}$ and $[\mathrm{M} / \mathrm{H}]$ across a sample of stars, as the line broadening due to low surface gravity must be compensated by a corresponding decrease in $[\mathrm{M} / \mathrm{H}]$. This effect is in turn compensated by a decrease in $T_{\text {eff }}$ that results in wider lines. ASPCAP's calibrations correct some of these effects, increasing $T_{\text {eff }}$ by around $+90 \mathrm{~K}$ and $[\mathrm{M} / \mathrm{H}]$ by $+0.027 \mathrm{dex}$ for stars with $[\mathrm{M} / \mathrm{H}]>-0.5 \mathrm{dex}$. The differences found when comparing our output $T_{\text {eff }}$ and $[\mathrm{M} / \mathrm{H}]$ with the ASPCAP values might be explained by the fact that, unlike their pipeline, our values for these parameters are not calibrated in any way.

Regarding the surface gravity differences between our work and ASPCAP results, we decided to run new syntheses with our method to three test stars, representative of the full sample. We fixed surface gravity to different values between $\log g=3.5 \mathrm{dex}$ and $\log g=5.0 \mathrm{dex}$, and compared the resulting changes in the other spectroscopic parameters. Since our pipeline and ASPCAP use similar methods for spectroscopic parameter derivation, by evaluating the magnitude of the differences we can conclude how problems in the derivation of $\log g$ via our method and via ASPCAP can affect other parameters. The results obtained for the three test stars are presented in Fig. 19. Comparing our test to a similar one with fixed $\log g$ values published in Tsantaki et al. (2018), we find that our results are similar to theirs, although with a larger dispersion in both $T_{\text {eff }}$ and $[\mathrm{M} / \mathrm{H}]$. The derived $T_{\text {eff }}$ can vary by up to $1000 \mathrm{~K}$, with a strong dependence on $\log g$. There is also a strong dependence on the output for $[\mathrm{M} / \mathrm{H}]$ as $\log g$ is fixed at different values, with differences varying up to 0.75 dex. The larger magnitude of these differences can be explained by the lower resolution of the analyzed spectra here compared to the spectra used by Tsantaki et al. (2018) to test the effects of fixing the $\log g$. We note that this interdependence of parameters reported for the spectral synthesis method is not relevant when using the ionization and excitation balance method (see also Mortier et al. 2013, 2014b for a further exploration of the effects fixing $\log g$ values can have on other spectroscopical parameters).

This test shows that performing syntheses with incorrect values for the surface gravity can result in errors across other parameters, and that fixing the $\log g$ can result in different parameters for the same star. Despite the strong effect that changing the $\log g$ can have on the other parameters, such as $T_{\text {eff }}$ and $[\mathrm{M} / \mathrm{H}]$, the fact that our uncorrected $\log g$ values are not very different from ASPCAP and optical values, and that our $T_{\text {eff }}$ and $[\mathrm{M} / \mathrm{H}]$ are also comparable, increases our confidence in our final set of parameters.

Another possible explanation for any discrepancies in the parameters may be just a difference in the way the parameters are minimized in our pipeline and in other codes. For example, the work of Jofré et al. (2014) explored the derivation of stellar parameters of the same stellar sample by multiple codes and demonstrated how the metallicities derived by Turbospectrum can be lower than values obtained with other methods. In addition, the recent work of Blanco-Cuaresma (2019) has shown how different spectral synthesis codes can derive different parameters with iSpec. This work focuses on optical wavelengths, but some differences, such as the ones described in Sect. 3.8 of that paper, "The one variable at a time experiment", are independent of the wavelength used. Further explorations of the output parameter space of Turbospectrum and other codes using NIR spectra are needed to know exactly what the explanation for these discrepancies might be.

Finally, even though our synthetic spectra provide a good match for the observed spectra, it does not mean we can fully trust the parameters or that they are better or worse than the ones published before in the literature. Since the observations are different from the ones in the optical, and the normalization is different from that used by ASPCAP as well, there is no way to conclusively determine the set of parameters that best characterize a particular star. We can state for certain that all the parameters shown in this paper were derived in a homogeneous way, using a uniform method and pipeline, and obtaining precise results. Therefore, any systematic error or bias will be present across the full sample.

\section{Conclusions}

Using synthetic spectra, we provided parameters for a sample of 3748 FGK main-sequence and subgiant stars observed with APOGEE. These parameters were derived using synthetic spectra generated by a pipeline using both iSpec and Turbospectrum. 
This method requires a functional and complete line list and complementing line mask for the $H$ band, which are included for future reference. The results were compared with multiple literature sources, and possible sources of discrepancies were explored.

Repeated iterations of Turbospectrum syntheses with similar spectra show how the method can provide consistent output parameters across multiple runs. The matching spectral synthesis and precise parameter determinations show that our pipeline can be a very powerful tool to synthesize stellar spectra and derive spectroscopic parameters for stars with $H$-band spectra. The fact that our synthesized spectra are a very close match to the observations (see Fig. 11) and that the average differences in derived parameters between our measurements and others are low (and below precision level in the specific case of comparison with optical values), means that this method can be a viable alternative in the future for homogeneous spectroscopic parameter derivation in the $H$ band, and may be expanded to other spectral types and spectral resolutions.

Even though this paper's focus is on FGK dwarfs, the most promising next step is to move towards colder stars and derive parameters for $\mathbf{M}$ dwarfs. Another paper exploring stars in this parameter space is currently in preparation.

Acknowledgements. This work was supported by FCT - Fundação para a Ciência e a Tecnologia through national funds (PTDC/FIS-AST/28953/ 2017, PTDC/FIS-AST/7073/2014, PTDC/FIS-AST/32113/2017, UID/FIS/ 04434/2013) and by FEDER - Fundo Europeu de Desenvolvimento Regional through COMPETE2020 - Programa Operacional Competitividade e Internacionalização (POCI-01-0145-FEDER-028953, POCI-01-0145-FEDER-016880, POCI-01-0145-FEDER-032113, POCI-01-0145-FEDER-007672). This work was supported by FCT/MCTES through national funds (PIDDAC) by grant UID/FIS/04434/2019. This research has made use of NASA's Astrophysics Data System. P. S. acknowledges the support by the Bolsa de Investigação $\mathrm{PD} / \mathrm{BD} / 128050 / 2016$. E. D. M. acknowledges the support by the Investigador FCT contract IF/00849/2015/CP1273/CT0003 and in the form of an exploratory project with the same reference. B. R.-A. acknowledges funding support from CONICYT PAI/CONCURSO NACIONAL INSERCION EN LA ACADEMIA CONVOCATORIA 2015 grant 79150050 and FONDECYT through grant 11181295.

\section{References}

Adibekyan, V. Z., Sousa, S., Santos, N., et al. 2012, A\&A, 545, A32

Albareti, F. D., Prieto, C. A., Almeida, A., et al. 2017, ApJS, 233, 25

Allende Prieto, C. 2011, Conversion from Vacuum to Standardair Wavelengths (Instituto de Astrofísica deCanarias)

Allende Prieto, C., Majewski, S., Schiavon, R., et al. 2008, Astron. Nachr., 329 1018

Alvarez, R., \& Plez, B. 1998, A\&A, 330, 1109

Artigau, É, Kouach, D., Donati, J. F., et al. 2014, in Ground-based and Airborne Instrumentation for Astronomy V, Int. Soc. Opt. Photon., 9147, 914715

Bean, J. L., Benedict, G. F., \& Endl, M. 2006, ApJ, 653, L65

Bensby, T., Feltzing, S., \& Oey, M. 2014, A\&A, 562, A71

Blanco-Cuaresma, S. 2019, MNRAS, 486, 2075

Blanco-Cuaresma, S., Soubiran, C., Heiter, U., \& Jofré, P. 2014, A\&A, 569, A111

Borucki, W. J., Koch, D. G., Basri, G., et al. 2011, ApJ, 736, 19

Bowen, I., \& Vaughan, A. 1973, Appl. Opt., 12, 1430

Bressan, A., Marigo, P., Girardi, L., et al. 2012, MNRAS, 427, 127
Brewer, J. M., Fischer, D. A., Valenti, J. A., \& Piskunov, N. 2016, ApJS, 225, 32

Brown, A., Vallenari, A., Prusti, T., et al. 2018, A\&A, 616, A1

Ciddor, P. E. 1996, Appl. Opt., 35, 1566

Cunha, K., Frinchaboy, P. M., Souto, D., et al. 2016, Astron. Nachr., 337, 922

De Silva, G. M., Freeman, K. C., Bland-Hawthorn, J., et al. 2015, MNRAS, 449, 2604

Delgado Mena, E., Tsantaki, M., Adibekyan, V. Z., et al. 2017, A\&A, 606, A94 Dotter, A., Chaboyer, B., Jevremović, D., et al. 2008, ApJS, 178, 89

Fleming, S. W., Mahadevan, S., Deshpande, R., et al. 2015, AJ, 149, 143

Garcia Pérez, A. E., Prieto, C. A., Holtzman, J. A., et al. 2016, AJ, 151, 144

Gazzano, J.-C., de Laverny, P., Deleuil, M., et al. 2010, A\&A, 523, A91

Gilmore, G., Randich, S., Asplund, M., et al. 2012, The Messenger, 147, 25

Girardi, L., Bressan, A., Bertelli, G., \& Chiosi, C. 2000, ApJS, 141, 371

Girardi, L., Bertelli, G., Bressan, A., et al. 2002, A\&A, 391, 195

Gray, D. F. 2005, The Observation and Analysis of Stellar Photospheres (Cambridge University Press)

Grevesse, N., Asplund, M., \& Sauval, A. 2007, Space Sci. Rev., 130, 105

Gunn, J. E., Siegmund, W. A., Mannery, E. J., et al. 2006, AJ, 131, 2332

Gustafsson, B., Edvardsson, B., Eriksson, K., et al. 2008, A\&A, 486, 951

Haywood, M. 2001, MNRAS, 325, 1365

Hernandez, J. G., \& Bonifacio, P. 2009, A\&A, 497, 497

Holtzman, J. A., Shetrone, M., Johnson, J. A., et al. 2015, AJ, 150, 148

Holtzman, J. A., Hasselquist, S., Shetrone, M., et al. 2018, AJ, 156, 125

Jofré, P., Heiter, U., Soubiran, C., et al. 2014, A\&A, 564, A133

Koleva, M., Prugniel, P., Ocvirk, P., Le Borgne, D., \& Soubiran, C. 2008, MNRAS, 385, 1998

Kurucz, R. L. 2005, Mem. Soc. Astron. It. Suppl., 8, 14

Latham, D., Brown, T., Monet, D., et al. 2005, Bull. Am. Astron. Soc., 37, 1340

Markwardt, C. B. 2009, ASP Conf. Ser., 411, 251

Mortier, A., Santos, N., Sousa, S., et al. 2013, A\&A, 558, A106

Mortier, A., Sousa, S., Adibekyan, V. Z., Brandão, I., \& Santos, N. 2014a, A\&A, 572, A95

Mortier, A., Sousa, S. G., Adibekyan, V. Z., Brandão, I. M., \& Santos, N. C. 2014b, A\&A, 572, A95

Newton, I. 1672, Philos. Trans., 6, 3075

Önehag, A., Heiter, U., Gustafsson, B., et al. 2012, A\&A, 542, A33

Origlia, L., Oliva, E., Baffa, C., et al. 2014, in Ground-based and Airborne Instrumentation for Astronomy V, Int. Soc. Opt. Photon., 9147, 91471E

Pepe, F., Mayor, M., Delabre, B.. et al. 2000, in Optical and IR Telescope Instrumentation and Detectors, Int. Soc. Opt. Photon., 4008, 582

Pepe, F., Lovis, C., Segransan, D., et al. 2011, A\&A, 534, A58

Pietrinferni, A., Cassisi, S., Salaris, M., \& Castelli, F. 2004, ApJ, 612, 168

Piskunov, N., Kupka, F., Ryabchikova, T., Weiss, W., \& Jeffery, C. 1995, A\&AS, 112,525

Plez, B. 2012, Astrophysics Source Code Library [record ascl:1205.004]

Quirrenbach, A., Amado, P., Caballero, J., et al. 2014, in Ground-based and Airborne Instrumentation for Astronomy V, Int. Soc. Opt. Photon., 9147, $91471 \mathrm{~F}$

Rocha-Pinto, H. J., \& Maciel, W. J. 1996, MNRAS, 279, 447

Rojas-Ayala, B., Covey, K. R., Muirhead, P. S., \& Lloyd, J. P. 2012, ApJ, 748, 93

Rowe, J. F., Bryson, S. T., Marcy, G. W., et al. 2014, ApJ, 784, 45

Santos, N. C., Sousa, S. G., Mortier, A., et al. 2013, A\&A, 556, A150

Shetrone, M., Bizyaev, D., Lawler, J. E., et al. 2015, ApJS, 221, 24

Soubiran, C., Le Campion, J.-F., Brouillet, N., \& Chemin, L. 2016, A\&A, 591, A118

Sousa, S., Santos, N., Mayor, M., et al. 2008, A\&A, 487, 373

Tsantaki, M., Sousa, S., Adibekyan, V. Z., et al. 2013, A\&A, 555, A150

Tsantaki, M., Andreasen, D. T., Teixeira, G. D. C., et al. 2018, MNRAS, 473, 5066

Valenti, J., \& Piskunov, N. 1996, A\&AS, 118, 595

Valenti, J. A., \& Fischer, D. A. 2005, ApJS, 159, 141

Wallace, L., Livingston, W., Hinkle, K., \& Bernath, P. 1996, ApJS, 106, 165

Zhao, G., Zhao, Y.-H., Chu, Y.-Q., Jing, Y.-P., \& Deng, L.-C. 2012, Res. Astron. Astrophys., 12, 723 
P. Sarmento et al.: Derivation of parameters for 3748 FGK stars using $H$-band spectra from APOGEE Data Release 14

\section{Appendix A: Air-vacuum wavelength}

APOGEE publishes its spectra in vacuum wavelength (Albareti et al. 2017), while the spectral lines in our line list are all in air wavelength. In order to use the lines in conjunction with APOGEE's spectra, they must be converted into the same format. Thus, a good conversion in the $H$-band spectra is essential to use iSpec's capabilities with APOGEE's spectra. This conversion is done using equations found in "Conversion from vacuum to standard air wavelengths" by Allende Prieto (2011). The equation used was

$$
\frac{\lambda_{0}-\lambda}{\lambda}=n-1=a+\frac{b_{1}}{c_{1}-1 / \lambda_{0}^{2}}+\frac{b_{2}}{c_{2}-1 / \lambda_{0}^{2}},
$$

Table A.1. Parameters used for air-vacuum conversion.

\begin{tabular}{lc}
\hline \hline Parameters & Value \\
\hline$a$ & 0.0 \\
$b_{1}$ & $5.792105 \times 10^{-2}$ \\
$b_{2}$ & $1.67917 \times 10^{-3}$ \\
$c_{1}$ & 238.0185 \\
$c_{2}$ & 57.362 \\
\hline
\end{tabular}

where $\lambda_{0}$ is given in $\mu \mathrm{m}$ and the parameters used were the ones in Table A.1 (Ciddor 1996). 
Appendix B: Additional synthesized spectra examples

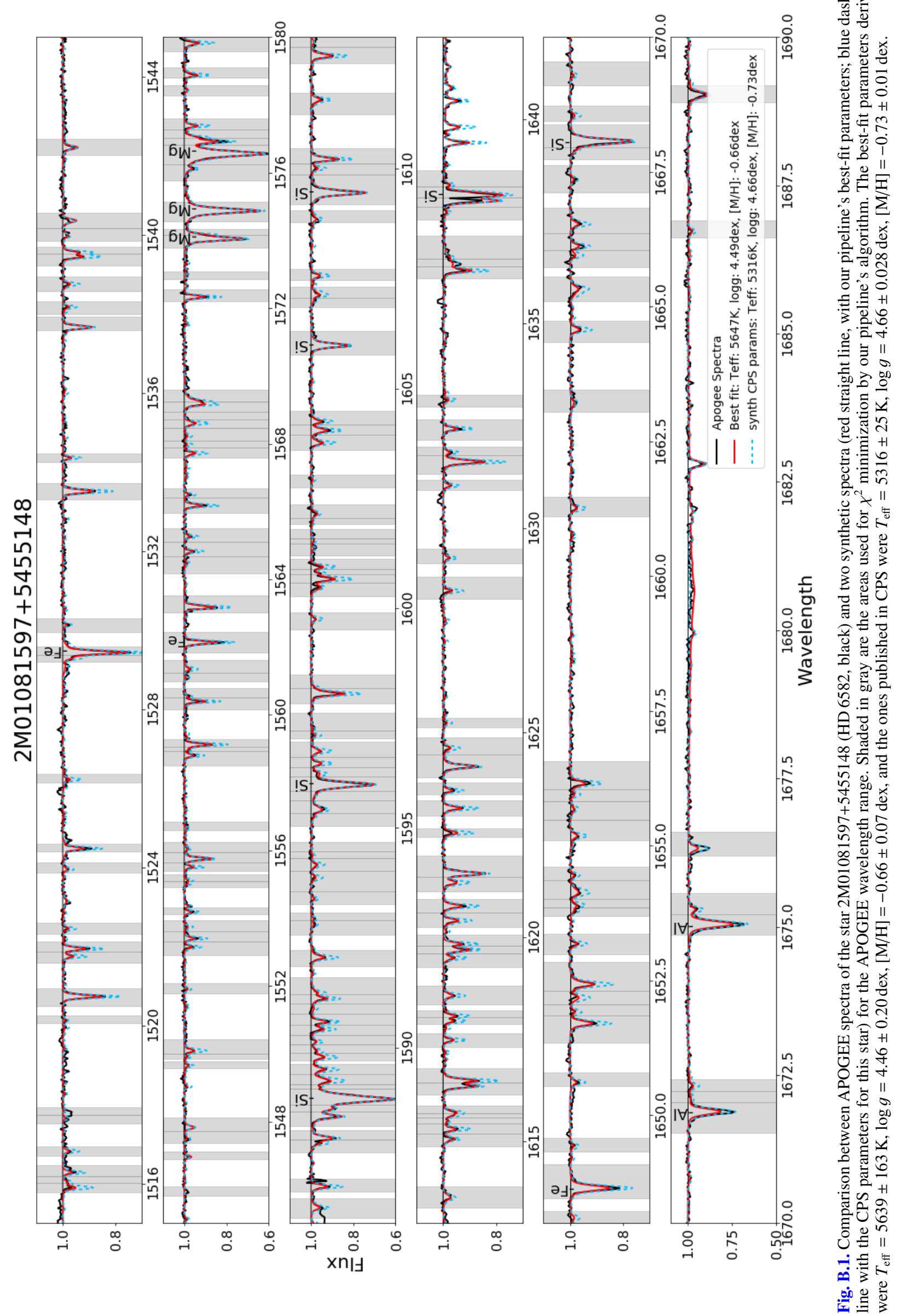




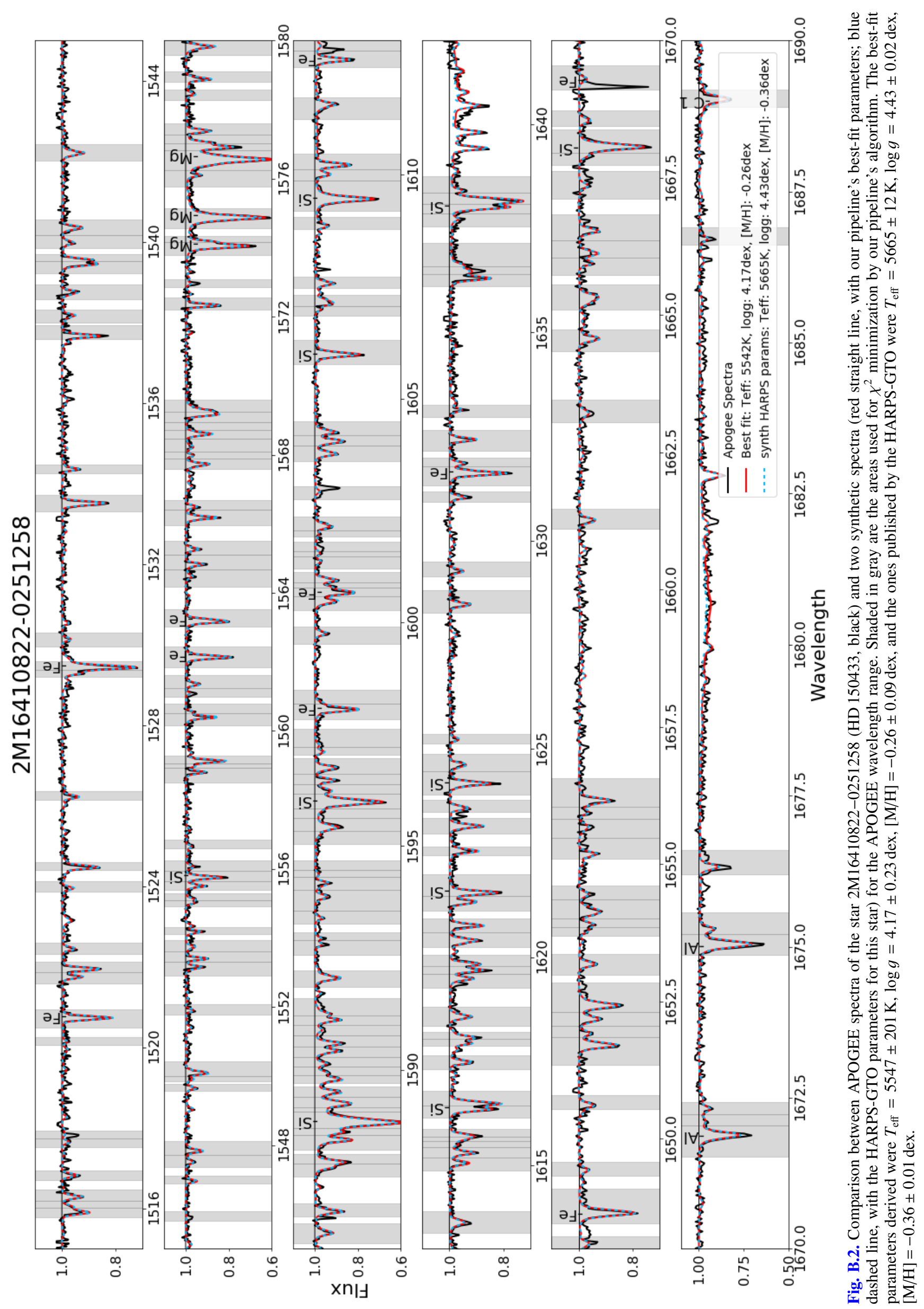




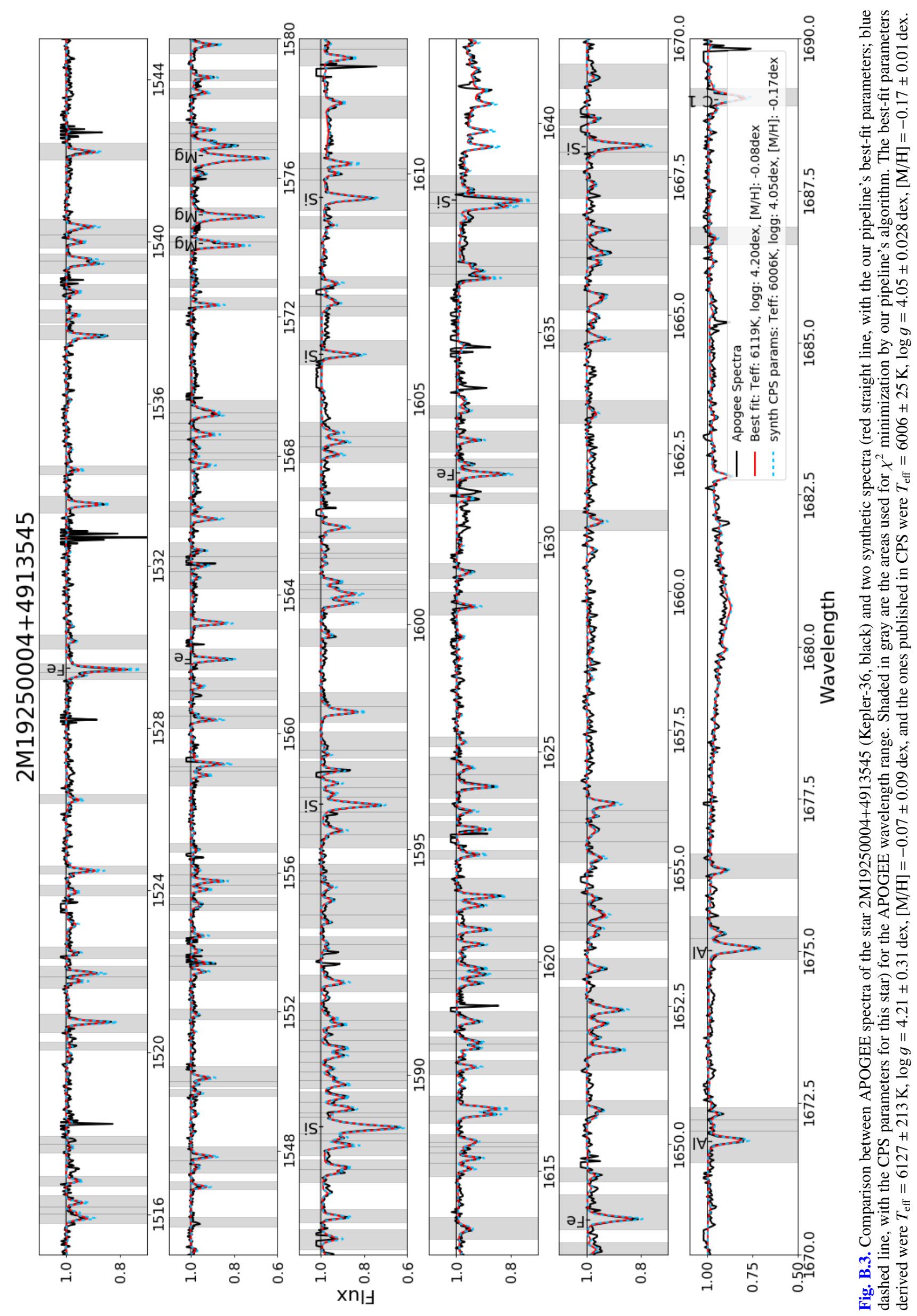


P. Sarmento et al.: Derivation of parameters for 3748 FGK stars using $H$-band spectra from APOGEE Data Release 14

Appendix C: Full results for stellar sample

Table C.1. Excerpt of the list of derived parameters for FGK stellar sample.

\begin{tabular}{|c|c|c|c|c|c|c|c|c|c|}
\hline APOGEE_ID & $T_{\text {eff }} \pm \Delta T_{\text {eff }}$ & $\log g \pm \Delta \log g$ & $\log g_{\text {cor }}$ & {$[\mathrm{M} / \mathrm{H}] \pm \Delta[\mathrm{M} / \mathrm{H}]$} & {$[\mathrm{Fe} / \mathrm{H}] \pm \Delta[\mathrm{Fe} / \mathrm{H}]$} & $v_{\text {mic }}$ & $v_{\mathrm{mac}}$ & $v \sin i$ & $\chi^{2}$ \\
\hline $2 \mathrm{M} 00012723+8520108$ & $5973 \pm 66$ & $4.24 \pm 0.07$ & 4.15 & $0.16 \pm 0.03$ & $0.11 \pm 0.1$ & 0.74 & 5 & 10.11 & 1.4 \\
\hline $2 \mathrm{M} 00015324+5634361$ & $6164 \pm 143$ & $4.15 \pm 0.2$ & 4.01 & $-0.15 \pm 0.06$ & $-0.23 \pm 0.1$ & 0.64 & 6.54 & 8.57 & 0.54 \\
\hline $2 \mathrm{M} 00072254+2627025$ & $4741 \pm 83$ & $3.18 \pm 0.11$ & 3.18 & $-0.03 \pm 0.04$ & $-0.15 \pm 0.1$ & 0.88 & 3.67 & 9.97 & 1.02 \\
\hline 2M00095611-0002296 & $6196 \pm 139$ & $4.47 \pm 0.15$ & 4.31 & $0.02 \pm 0.05$ & $-0.05 \pm 0.1$ & 0.21 & 6.54 & 8.48 & 1.41 \\
\hline $2 \mathrm{M} 00100176+0201021$ & $6142 \pm 115$ & $4.26 \pm 0.16$ & 4.12 & $0.09 \pm 0.04$ & $0.02 \pm 0.1$ & 0.67 & 6.11 & 8.99 & 1.46 \\
\hline $2 \mathrm{M} 00125570-1441121$ & $5730 \pm 111$ & $4.3 \pm 0.12$ & 4.27 & $0.04 \pm 0.05$ & $0 \pm 0.1$ & 0.65 & 4 & 9.3 & 0.44 \\
\hline 2M00135646-1439234 & $5927 \pm 127$ & $4.33 \pm 0.19$ & 4.25 & $-0.15 \pm 0.05$ & $-0.21 \pm 0.1$ & 0.16 & 5.08 & 8.3 & 1.46 \\
\hline 2M00153822-1516023 & $6181 \pm 145$ & $4.52 \pm 0.15$ & 4.37 & $0.09 \pm 0.06$ & $0.04 \pm 0.1$ & 0.52 & 6.35 & 10.49 & 1.7 \\
\hline 2M00162970-1456483 & $6144 \pm 122$ & $4.36 \pm 0.19$ & 4.22 & $0.11 \pm 0.05$ & $0.06 \pm 0.1$ & 0.68 & 6.08 & 10.61 & 0.48 \\
\hline 2M00164203-1533376 & $5852 \pm 140$ & $4.23 \pm 0.2$ & 4.17 & $-0.18 \pm 0.06$ & $-0.27 \pm 0.1$ & 0.36 & 4.76 & 8.23 & 0.48 \\
\hline 2M00170412-1459180 & $5598 \pm 106$ & $4.27 \pm 0.13$ & 4.27 & $-0.01 \pm 0.05$ & $-0.04 \pm 0.1$ & 0.88 & 3.62 & 9.98 & 0.56 \\
\hline
\end{tabular}

Notes. Uncertainties on derived parameters are estimated by iSpec. iSpec output parameters are indicated by the letter O. Literature parameters are indicated by the letter L. Full list available at the CDS. 